\title{
Energetic electrons generated during solar flares
}

\author{
Gottfried Mann $\dagger$ \\ Leibniz-Institut für Astrophysik Potsdam, An der Sternwarte 16, 14482 Potsdam, Germany
}

(Received 22 May 2015; revised 28 September 2015; accepted 29 September 2015)

The Sun is a giant particle accelerator. During solar flares, magnetic field energy stored in the corona is suddenly released and transferred to local heating of the coronal plasma, mass motions (e.g. jets) and the generation of energetic particles, i.e. electrons, protons and heavy ions. Basically, a flare occurs as a local enhancement of the emission of electromagnetic radiation from the radio up to the $\gamma$-ray range on the Sun. That indicates the production of energetic electrons during flares. NASA's RHESSI mission has the aim to investigate electron acceleration processes by studying the Sun's X-ray and $\gamma$-ray emission with high spatial, temporal and spectral resolution, i.e. by means of imaging spectroscopy. A substantial part of the energy released during a flare is carried by these energetic electrons. Apart from them, solar energetic particles, i.e. protons and heavy ions, and coronal mass ejections play an important role in the energy budget of a flare. Here, we focus on electron acceleration. The way in which $10^{36}$ electrons are accelerated up to energies beyond $30 \mathrm{keV}$ is one of the open questions in solar physics. A flare is considered as the manifestation of magnetic reconnection in the solar corona. Which mechanisms lead to the production of energetic electrons in the magnetic reconnection region is discussed in this paper. Two of them are described in more detail.

\section{Introduction}

The Sun is an active star. This manifests itself not only in the well-known sunspots with related 11-year cycle, but also in flares, coronal mass ejections (CMEs) and solar energetic particle (SEP) events. Basically, flares are defined as a sudden and local enhancement of the emission of electromagnetic radiation on the Sun's disc (Priest 1981). The electromagnetic radiation of a flare covers a broad spectrum from the radio up to the $\gamma$-ray range. For example, figure 1 shows an extreme ultraviolet (EUV) image of the Sun during the flare on 28 October 2003. It was a >X17-class flare of the GOES classification according to the 1-8 $\AA$ soft X-ray peak intensity, and hence one of the hugest flares ever observed. For comparison, the famous Carrington event on 1 September 1859 can be estimated to be an X45 $( \pm 5)$ flare (Cliver \& Svalgaard 2004; Cliver \& Dietrich 2013). A flash of EUV emission is seen in the middle of the southern hemisphere of the Sun in figure 1. For the same flare, figure 2 shows the fluxes of the $\gamma$-ray and hard X-ray radiation (top) as well as the simultaneous dynamic radio spectrum (bottom) in the frequency range $200-400 \mathrm{MHz}$. Inspection of figure 2 reveals that the flare starts with an enhanced radio emission near $300 \mathrm{MHz}$. The radio

$\dagger$ Email address for correspondence: GMann@aip.de 


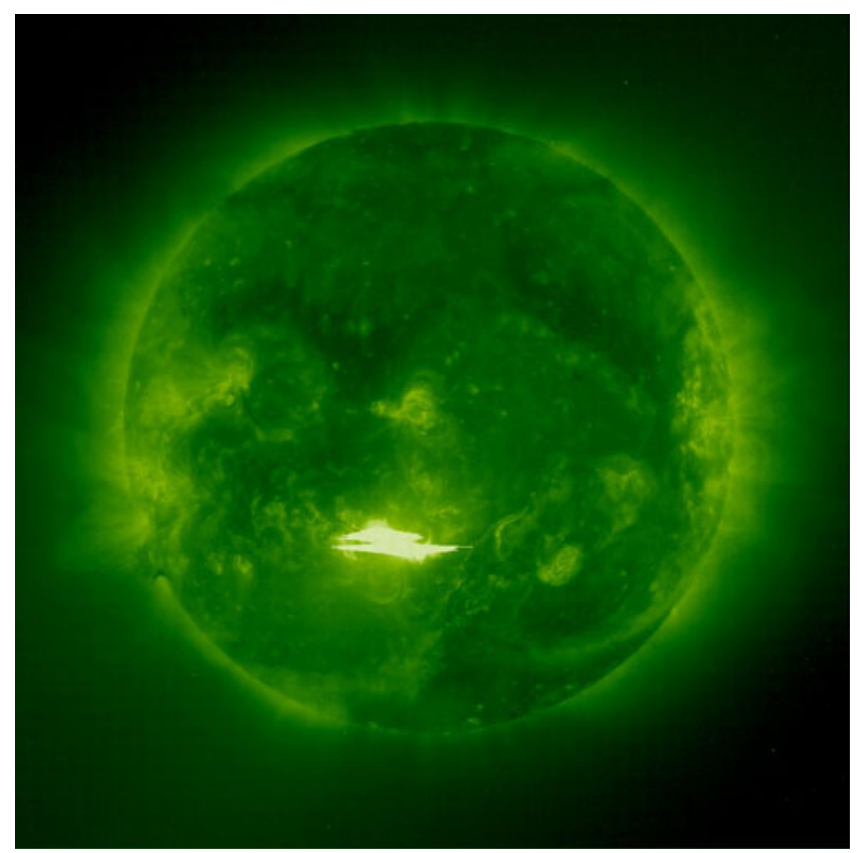

FIGURE 1. EUV image of the Sun recorded at $165 \AA$ with the EIT instrument onboard ESA/NASA's SoHO spacecraft on 28 October 2003.

emission rapidly extends over a broad spectrum. Simultaneously, the $\gamma$-ray emission strongly increases in the energy range 7.5-10 MeV for a few seconds. That indicates the production of electrons with energies beyond $10 \mathrm{MeV}$. The hard X-ray emission is also enhanced but for a longer period in comparison to the $\gamma$-ray radiation.

It is widely accepted that, during a flare, stored magnetic field energy is suddenly released and transferred into local heating of the coronal plasma, mass motions (e.g. jets; Shibata et al. 1992), enhanced emission of electromagnetic radiation, and enhanced fluxes of energetic particles, i.e. electrons, protons and heavy ions (Heyvaerts 1981). Thus, the Sun is a giant particle accelerator (Lin 1974; Reames, Barbier \& Ng 1996; Klein \& Trottet 2001). Emslie et al. (2012) studied the global energetics of a large sample of solar eruptive events. They found that the energy contents of flare-accelerated electrons and ions are approximately comparable. Mostly, the kinetic energy of the CMEs is the major part of the whole energy budget. The energetic electrons are of special interest, since they can carry a substantial part of the energy released in a flare (Lin \& Hudson 1971, 1976; Emslie et al. 2004). Therefore, we focus in this paper on electron acceleration during solar flares.

Presently, flares are widely understood in terms of the so-called standard or CSHKP model (Carmichael 1964; Sturrock 1966; Hirayama 1974; Kopp \& Pneuman 1976). Figure 3 shows schematically the scenario of this model. According to this model, in the corona, a prominence as a closed magnetic field structure is destabilized due to its photospheric footpoint motions and rises upwards. Hence, the underlying magnetic field lines are stretched. That leads to the formation of a current sheet. If the current within this sheet exceeds a critical value, the resistivity is suddenly increased by plasma wave excitation due to various instabilities (Treumann \& Baumjohann 1997). Then, magnetic reconnection can take place there. The region of enhanced resistivity 


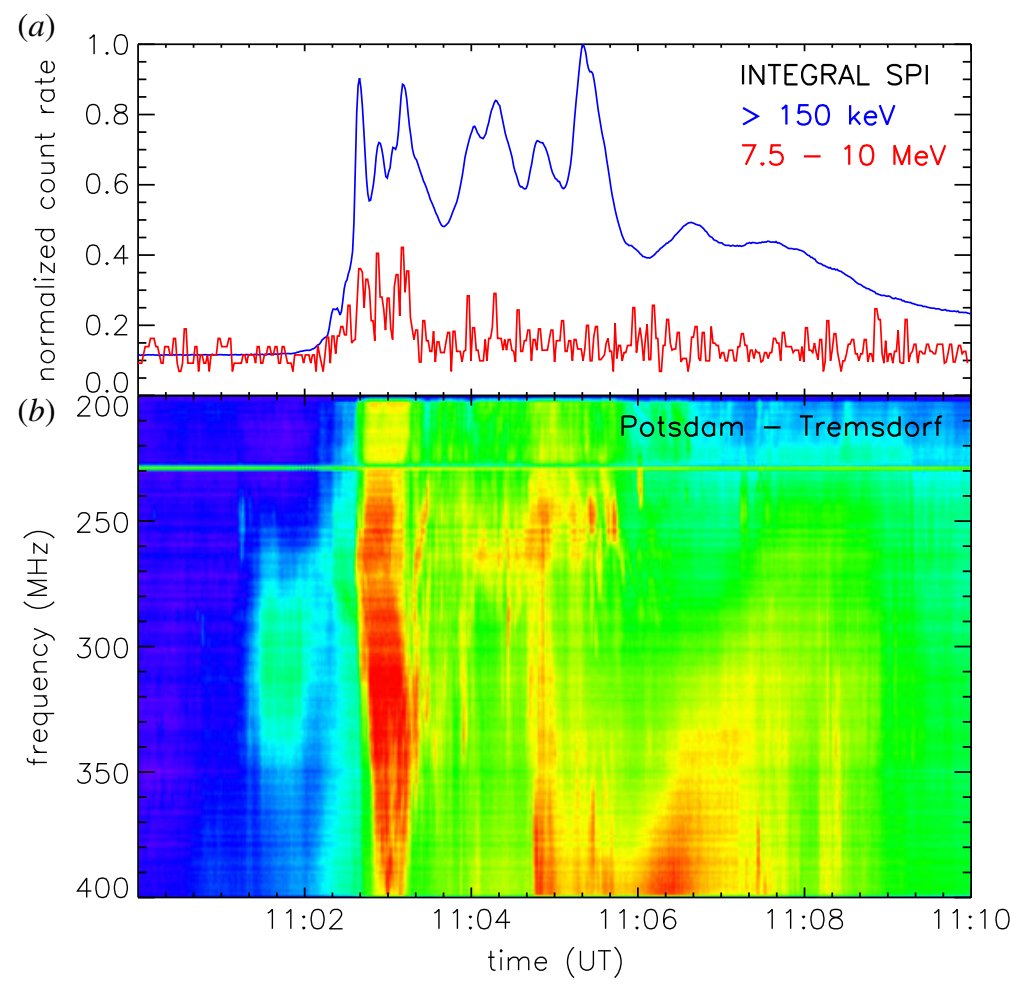

FIGURE 2. (a) Fluxes of $\gamma$-ray radiation in the range 7.5-10 MeV (red) and hard X-ray radiation (blue) in the energy range $>150 \mathrm{keV}$ as well as $(b)$ the simultaneous dynamic radio spectrum in the frequency range $200-400 \mathrm{MHz}$ for the solar event on 28 October 2003 (see figure 5 in Mann, Warmuth \& Aurass 2009).

is called the diffusion region. Because of the strong curvature of the magnetic field lines in the vicinity of the diffusion region, the slowly inflowing plasma shoots away from the reconnection site as oppositely directed hot jets. The inflow region is separated from the outflow one by pairs of slow-mode standing shocks. If the speed of the outflow jet is super-Alfvénic and the jet penetrates into the surrounding coronal plasma, a fast-mode shock, which is called a termination shock (TS) is established (see figure 3). This scenario is supported by both numerical simulations (Forbes 1986; Yokoyama \& Shibata 1994) (see Priest \& Forbes 2002 for a review) and observations (Masuda et al. 1994; Tsuneta 1996).

The discovery of a loop-top hard X-ray source with the Yohkoh satellite by Masuda et al. (1994) can be considered as evidence of magnetic reconnection taking place above the flare loop, i.e. in the corona. Electrons are accelerated at the magnetic reconnection site, travel down and emit hard X-rays above the flare loop. The CSHKP model as described is basically two-dimensional. Thus, a three-dimensional extension is necessary and recently done, for instance, by Shibata et al. (1995), Priest \& Forbes (2002), Aulanier, Janvier \& Schmieder (2012). In three dimensions, new magnetic field topologies such as the null, the spine curve, the fan surface and the separator becomes possible. Since electric currents occur at these places, magnetic reconnection can take place there (see the review by Priest \& Forbes (2002) for further discussion). 


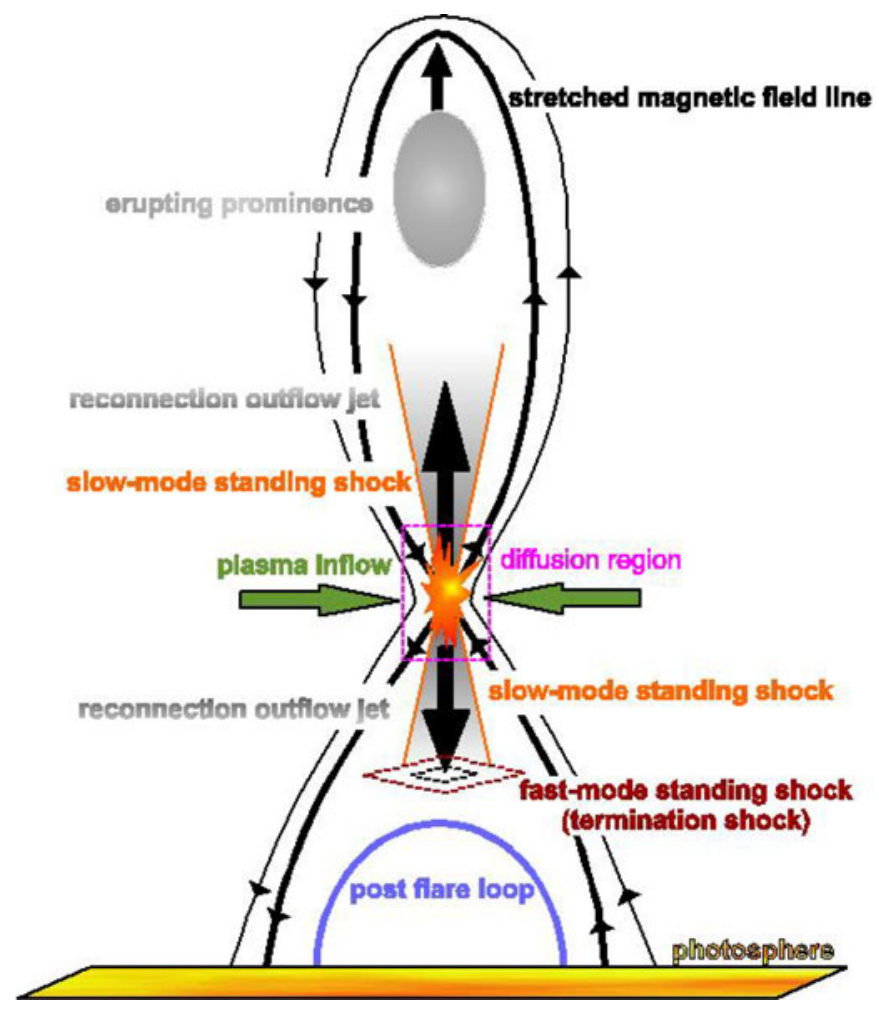

FIGURE 3. Sketch of the standard flare model (see figure 1 in Mann et al. 2009).

According to this standard flare model, the primary energy release and the electron acceleration take place in the corona. That is supported by several observations:

(i) Flares are often accompanied by non-thermal radio emission (see Fletcher et al. 2011; White et al. 2011 for recent reviews) indicating the occurrence of energetic electrons in the corona. The Sun's non-thermal radio radiation in the decimetre and metre wave range is considered to be generated by plasma emission. Energetic electrons excite high-frequency plasma waves, e.g. Langmuir waves, which convert by nonlinear processes into escaping radio waves. Hence, the radio waves are emitted near the local electron plasma frequency and/or its harmonics (Melrose 1985). Just that is seen in figure 2. Already at 11:01:20 UT, i.e. before the start of the strong increase of $\gamma$-ray emission at 11:02:20 UT, an enhanced radio emission occurs around $300 \mathrm{MHz}$. Assuming fundamental plasma emission, it corresponds to an electron number density of $\approx 10^{9} \mathrm{~cm}^{-3}$. That is a typical value for the corona (Koutchmy 1994). Thus, the dynamic radio spectrum in figure 2 reveals that the primary energy release started in the corona.

Imaging of the hard X-ray and radio sources with RHESSI and the Nancay Radioheliograph for the flare on 20 February 2002 reveal a close relationship between the energetic electrons producing both the hard X-ray and radio radiation (Vilmer et al. 2002). Furthermore, Reid, Vilmer \& Kontar (2011) and Reid, Vilmer \& Kontar (2014) found a close relationship between type III radio bursts and the associated signatures in the hard X-ray radiation. That allows determination of the height and vertical extent of the acceleration region to be 
$\approx 50 \mathrm{Mm}$ and $10 \mathrm{Mm}$, respectively (Reid et al. 2011, 2014). Type III radio bursts are considered as radio signatures of beams of energetic electrons travelling along magnetic field lines through the corona (Suzuki \& Dulk 1985).

(ii) The soft and hard X-ray observations of flares by Yohkoh reveal a double source structure in terms of hard X-ray footpoints, a soft X-ray source in the flare loops, and a hard X-ray source above the top of the loop (Masuda et al. 1994). By means of the time-of-flight method developed by Aschwanden et al. (1996), the location of the electron acceleration site has been determined in the vicinity of the loop-top hard X-ray sources. Since RHESSI provides for the first time imaging spectroscopy of solar flares in both the hard X-ray and $\gamma$-ray range, RHESSI observations allow one to discriminate between chromospheric and coronal X-ray and $\gamma$-ray sources and to determine the X-ray spectrum at different locations. That was demonstrated for the solar event on 23 July 2002 by Emslie et al. (2003). They found that there are four hard X-ray sources, namely two footpoint sources near the chromosphere, a coronal source and a middle source with a spectrum similar to those of the footpoint sources. The footpoint sources are localized in oppositely polarized magnetic field structures. Therefore, they are considered as the two foots of a coronal loop (Krucker, Hurford \& Lin 2003). Battaglia \& Benz (2006) considered several flares with three or more hard $\mathrm{X}$-ray sources in order to study the evolution of X-ray spectra of the different sources. The spectra show a so-called soft-hard-soft behaviour, whereas the coronal sources are always softer.

(iii) As mentioned above, the hard X-ray sources during flares can be divided into the chromospheric footpoint sources and the coronal loop-top sources. The X-ray radiation of the footpoint sources are generated via thick-target emission. The $\mathrm{X}$-ray radiation emitted from coronal sources is generated by a combination of thermal and thin-target bremsstrahlung (Simões \& Kontar 2013). Studying several flares, Simões \& Kontar (2013) found that the rate of producing highly energetic electrons is higher by a factor $2-8$ in the coronal sources than in the footpoint sources.

(iv) Krucker, Hannah \& Lin (2007) and Krucker \& Lin (2008) reported on events where the chromospheric hard X-ray sources were occulted by the limb. In these cases, there are two components in the X-ray emission, a thermal one at low energies (i.e. $\approx 18 \mathrm{keV}$ ) with a gradual time profile and a second one with a rapid time variation and power-law spectra. The centroid position of the sources are frequently co-spatial. These observations 'present evidence for thin-target emission from flare-accelerated electrons in the corona ... implying a coronal acceleration region' (Krucker et al. 2007). Furthermore, there are a few events of flares in which RHESSI measured $\gamma$-ray sources in both the footpoints and the tops of the loops, indicating flare-accelerated electrons with energies up to $>1 \mathrm{MeV}$. These electrons stay long enough in the corona to produce $\gamma$-ray radiation (Krucker et al. 2008b).

The radio, hard X-ray and $\gamma$-ray radiation of flares has sources in the corona, leading to the conclusion that the primary energy release and the associated electron acceleration take place in the corona, supporting the standard (or CSHKP) model. RHESSI as an imaging spectrometer opened for the first time a direct diagnostic of electron acceleration during flares (see further discussions in the review paper by Krucker et al. (2008a)). 
In this paper, we will focus on the generation of energetic electrons in the solar atmosphere during flares. A summary of the observational results concerning flareaccelerated electrons is given in $\S 2$. There are several mechanisms for explaining the production of energetic electrons during flares, as briefly summarized in $\S 3$. Two special electron acceleration mechanisms, namely at the TS and the slow-mode shocks in the reconnection region, are discussed in more detail. It is emphasized that energetic electrons are produced not only by flares but also during quiet solar conditions. For example, Lin et al. (1996) reported on electron spectra in the solar wind during quiet periods, i.e. without any flare activities. These spectra have a pronounced suprathermal tail up to energies of $\approx 100 \mathrm{keV}$. This paper will be closed with a summary (see $\S 4$ ).

\section{Observational results}

The flare-enhanced emission of electromagnetic radiation indicates the generation of energetic electrons during flares. In the framework of the standard model (see § 1), the electrons are regarded as accelerated in the vicinity of the magnetic reconnection region. Since these electrons can emit both radio and X-ray radiation, observations of the Sun's radio and X-ray range gives information for a better understanding of electron acceleration during flares.

\subsection{Radio observations}

The Sun's radio emission in the decimetre and metre range is generally thought to be plasma emission, i.e. its emission occurs near the local electron plasma frequency and/or its harmonics. Since the electron plasma frequency is proportional to the square root of the electron number density, the higher and lower frequencies are emitted in the lower and higher corona, respectively, because of the gravitational density stratification of the solar atmosphere. Hence, a radio source moving upwards in the corona would appear as a drift from high to low frequencies in dynamic radio spectra. Sometimes, so-called pairs of forward and reverse drift bursts (McLean 1985) occur during flares. In dynamic radio spectra, they appear as stripes of enhanced radio emission. They start at a frequency $f_{s t}$ and rapidly drift towards higher and lower frequencies. An example is seen in figure 2 at the beginning of the event. They are interpreted as the radio signature of electron beams that are initially produced in the vicinity of the reconnection region and subsequently travel along magnetic field lines towards both the lower and higher corona (Suzuki \& Dulk 1985). Hence, the radio radiation of these electrons emanates immediately from the vicinity of the magnetic reconnection region. In the framework of the standard model the start frequency $f_{s t}$ is roughly the plasma frequency at the X-point (i.e. diffusion region) of the magnetic reconnection region.

In order to relate the emission frequency to the height in the corona, a Newkirk (1961) model

$$
N_{e}(r)=\alpha N_{0} 10^{4.32 R_{\odot} / r},
$$

where $N_{e}$ is the electron number density, $N(0)=4.2 \times 10^{4} \mathrm{~cm}^{-3}, R_{\odot}$ is the radius of the Sun and $r$ is the distance from the centre of the Sun, is adopted as a coronal density model. It corresponds to a barometric density stratification with a temperature of $1.4 \times 10^{6} \mathrm{~K}$ (Mann et al. 1999). White-light scattering measurements of the corona by Koutchmy (1994) reveal that $\alpha=4$ represents an appropriate density model above active regions. In this case, the maximum electron number density at the bottom of the 
corona (i.e. $r=R_{\odot}$ ) is $3.51 \times 10^{9} \mathrm{~cm}^{-3}$, corresponding to an electron plasma frequency $f_{p e}=532 \mathrm{MHz}$ (here $f_{p e}=\omega_{p e} / 2 \pi, \omega_{p e}=\left(4 \pi \mathrm{e}^{2} N_{e} / m_{e}\right)^{1 / 2}, e$ is the elementary charge and $m_{e}$ is the electron mass).

Of course, there are other coronal density models (e.g. Saito et al. 1970; Saito, Poland \& Munro 1977; Leblanc, Dulk \& Bougeret 1998; Mann et al. 1999). They describe the behaviour of the density at different parts of the corona, such as loops, streamers and coronal holes, as well as, partly, the transition from the corona into interplanetary space. The Newkirk (1961) model agrees well with the observations in the corona as emphasized by Koutchmy (1994) - see also figure 2 and the related discussions in Warmuth \& Mann (2005). Therefore, it can be used as an appropriate model for the inner corona, where flares occur.

Aschwanden et al. (1995a) presented a sample of 30 pairs of such forward and reverse drift bursts with a start frequency $f_{s t}$ in the range $272-955 \mathrm{MHz}$. If all of them with $f_{s t}<532 \mathrm{MHz}$ and $f_{s t}>532 \mathrm{MHz}$ are considered to be generated by fundamental and harmonic emission, respectively, $f_{s t}=360 \mathrm{MHz}$ is the mean value for this sample. Hence, an electron number density of $1.61 \times 10^{9} \mathrm{~cm}^{-3}$ is a typical value at the $\mathrm{X}$ point. According to a fourfold Newkirk (1961) model (i.e. $\alpha=4$ in (2.1)), such an electron number density is located at a height of $60 \mathrm{Mm}$ above the photosphere (Mann \& Warmuth 2011).

In the corona, the magnetic field cannot be measured directly as in the photosphere by the Zeeman effect. But the coronal magnetic field can be determined from observations by employing the model assumptions. By means of this method, Dulk \& McLean (1978) presented a model of the radial behaviour of the magnetic field above active region in the corona:

$$
B(r)=0.5 G \times\left(\frac{r}{R_{\odot}}-1\right)^{-1.5} .
$$

Of course, in reality the magnetic field topology is quite complex, especially above active regions, where we have open and closed magnetic field structures in a close neighbourhood. Warmuth \& Mann (2005) considered the behaviour of the magnetic field above an active region, i.e. the total magnetic field is a superposition of the magnetic field of the active region and that of the quiet Sun. Hence, there are locations of maximum and minimum of the magnetic field strength. But, in general, the Dulk \& McLean (1978) model can be considered as a good approximation for the behaviour of the magnetic field in the corona. According to this model, a magnetic field of $20 \mathrm{G}$ is expected at a height of $60 \mathrm{Mm}$ above the photosphere.

As a result, radio observations in terms of pairs of forward and reverse drift bursts provide an electron number density of $1.61 \times 10^{9} \mathrm{~cm}^{-3}$ and a magnetic field of $20 \mathrm{G}$ as typical values at the $\mathrm{X}$-point of the magnetic reconnection region. Of course, these values vary from case to case. Thus, the values given here should be considered as typical ones in a statistical sense.

\subsection{X-ray observations}

The energetic electrons originally produced in the vicinity of the magnetic reconnection region travel along the magnetic field line towards the dense chromosphere, where they emit X-ray radiation via bremsstrahlung (Brown 1971). But these electrons can temporarily be stored in the corona, leading to coronal X-ray sources (Krucker et al. 2010). Note that, in contrast to the radio radiation, the X-ray radiation is not emitted 


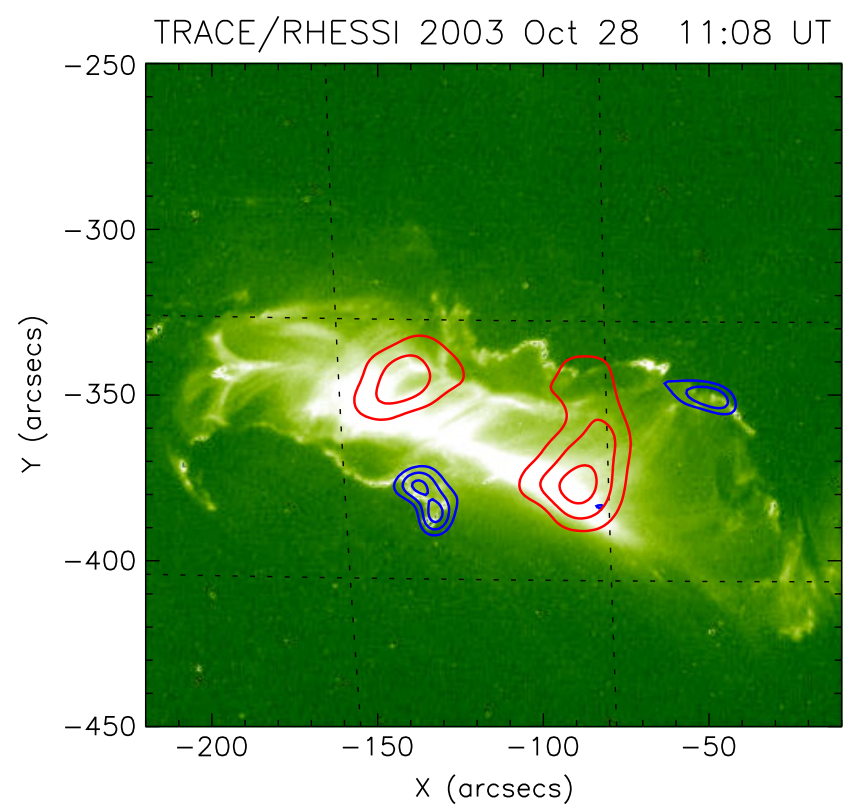

FIgURE 4. The soft (6-12 keV) (red contours) and hard (100-300 keV) (blue contours) $\mathrm{X}$-ray sources of the solar event (flare) on 28 October 2003. The X-ray sources are overlaid on the simultaneous TRACE EUV image at $195 \AA$ of the Sun.

immediately from the reconnection site. Figure 4 shows the location of the soft and hard X-ray sources for the event on 28 October 2003. It reveals that the soft X-ray source is located at the top of the loop magnetically connecting the two flare ribbons of opposite magnetic polarity. In contrast to that, there is a double structure of hard X-ray sources, which are located near the loop footpoints. The hard X-ray sources have a typical size of $10 \mathrm{Mm} \times 8 \mathrm{Mm}$ corresponding to an area $A_{\text {source }}=8 \times 10^{17} \mathrm{~cm}^{2}$ (Warmuth, Mann \& Aurass 2009b).

The corresponding hard X-ray spectrum measured by RHESSI (Lin et al. 2002) is shown in figure 5. Here, the photon spectra were folded with the full detector response matrix. The correction of decimation (Smith et al. 2002), the gain effect and the photospheric albedo (Kontar et al. 2006) were taken into account - see also the detailed description of the employed method in Warmuth et al. (2009a). The $\mathrm{X}$-ray spectrum of figure 5 is a typical flare spectrum. It consists of a thermal and non-thermal component. The non-thermal one is strongly pronounced above $28 \mathrm{keV}$ and extends beyond $300 \mathrm{keV}$. The thermal component corresponds to a temperature of $40.5 \times 10^{6} \mathrm{~K}$. The photon spectrum can be converted into a differential injected electron flux by means of the forward-fitting method developed by Holman et al. (2003), taking into account the photospheric albedo - see the detailed description of the method in Warmuth et al. (2009a) (see figure 5b). Of course, the conversion of the photon spectrum into the differential injected electron flux can be influenced by a non-uniform ionization of the target, albedo, Langmuir waves, return current, etc. All these effects can lead to changes in the resulting differential injected electron fluxes - see the discussion in the reviews by Holman et al. (2011) and Kontar et al. (2011). Note that the high-energy-resolution data from RHESSI have permitted the first reliable model-independent conversion of observed X-ray spectra into electron 

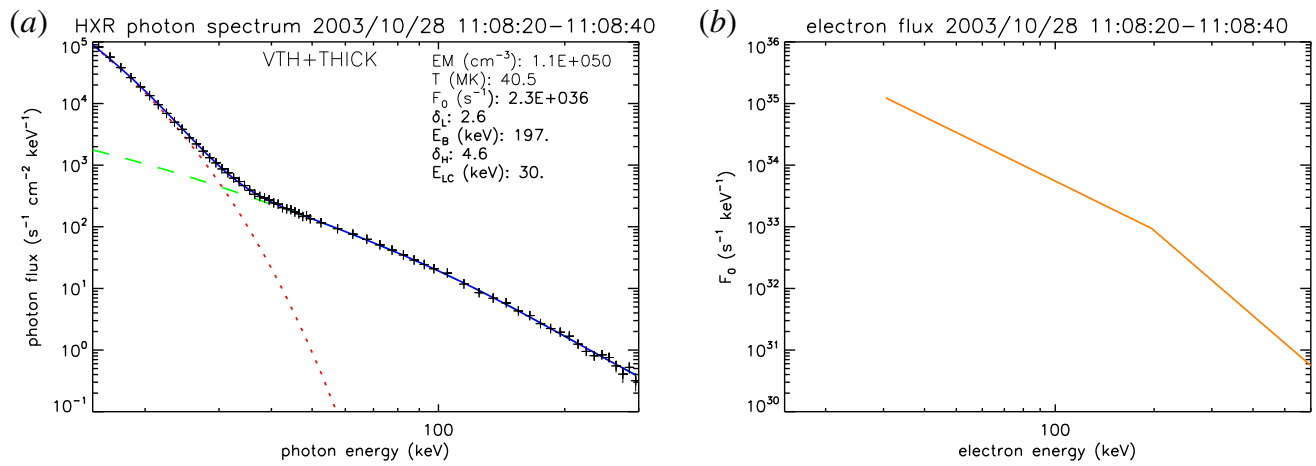

FIGURE 5. (a) The X-ray photon spectrum as recorded by RHESSI during the period 11:08:20-11:08:40 UT on 28 October 2003. (b) The differential electron flux derived from the photon spectrum in $(a)$ by means of the forward-fitting method developed by Holman et al. (2003).

spectra (Massone et al. 2003; Piana et al. 2003). For the example under discussion, the conversion of the photon spectrum provides a total flux $F_{e}=1.6 \times 10^{36} \mathrm{~s}^{-1}$ for electrons with an energy $>28 \mathrm{keV}$. It is related to an energy flux $P_{e}=1.5 \times 10^{22} \mathrm{~W}$ (Warmuth, Mann \& Aurass 2007). The ratio $P_{e} / F_{e}=59 \mathrm{keV}$ is the mean value of the energy of the electrons with energies $>28 \mathrm{keV}$ as produced during this special flare.

In order to study this subject in more detail, a sample of nine X-class GOES flares was chosen to calculate the differential electron flux during peak periods of hard X-ray fluxes. This sample covers flares in the GOES class range X1.3-X17.2, i.e. they are large flares. The differential fluxes calculated from the photon spectra by means of the forward-fitting method (Holman et al. 2003) can be described in terms of a broken power law

$$
j_{(E)}=j_{B}\left(\frac{E}{E_{B}}\right)^{-\delta},
$$

with $\delta=\delta_{L}$ for $E_{l c} \leqslant E \leqslant E_{B}$ and $\delta=\delta_{H}$ for $E_{B} \leqslant E$. Here, $E_{l c}$ is the so-called lowenergy cut-off, $E_{B}$ is the energy where the power law breaks, and $j_{B}$ is the differential flux at $E=E_{B}$. The logarithmic mean of all nine differential fluxes provides: $j_{B}=2.8 \times$ $10^{14} \mathrm{~cm}^{-2} \mathrm{~s}^{-1} \mathrm{keV}^{-1}, E_{l c}=28 \mathrm{keV}, E_{B}=148 \mathrm{keV}, \delta_{l}=4.3$ and $\delta_{H}=4.7$. These values can be considered as typical ones for large flares. (For more details, see Warmuth et al. (2009b) and Mann \& Warmuth (2011).) Inserting these values into (2.3), the flux $F_{X C F}$ of electrons with energies $>28 \mathrm{keV}$ is found to be $3.1 \times 10^{18} \mathrm{~cm}^{-2} \mathrm{~s}^{-1}$. These electrons carry an energy flux $P_{X C F}=1.2 \times 10^{20} \mathrm{keV} \mathrm{cm} \mathrm{cm}^{-2} \mathrm{~s}^{-1}$. Then, $P_{e} / F_{e}=39 \mathrm{keV}$ is the mean energy of the electrons accelerated up to an energy $>28 \mathrm{keV}$ during large flares. Note once again that these results are found for X-class flares, which are the largest ones.

\subsection{Plasma conditions in the flare region}

Figure 6 shows the geometry of the reconnection region in the corona according to the standard model (see figure 3). From solar radio data, i.e. pairs of forward and reversed drift bursts, the location of the X-point has already been found to be $h_{X}=60 \mathrm{Mm}$. Loops have typical radii of $10 \mathrm{Mm}$ (Aschwanden 2002). The height $h_{a c c}$ of the acceleration region is determined to be $20 \mathrm{Mm}$ (Mann \& Warmuth 2011) 


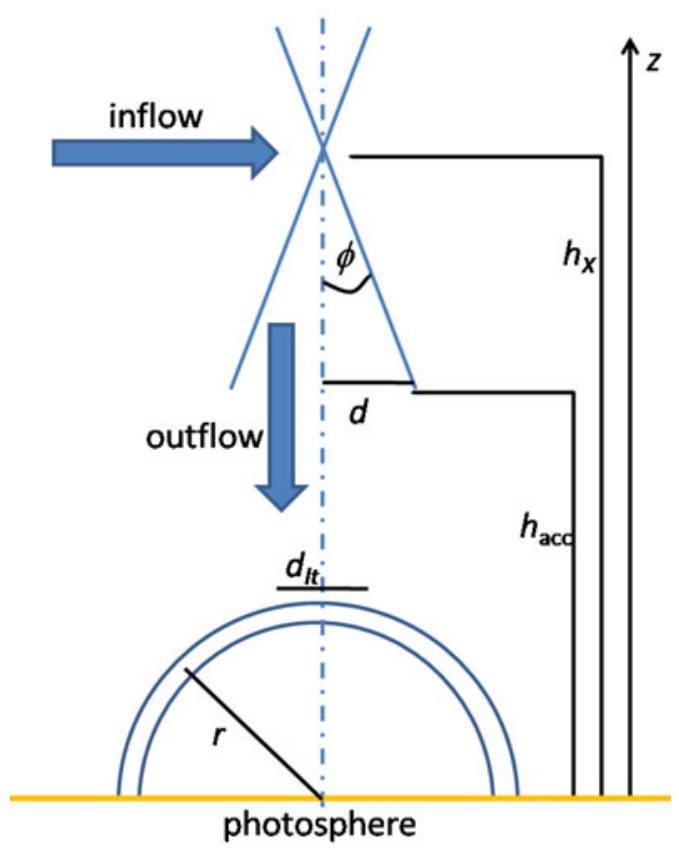

FIGURE 6. Geometry of the reconnection region according to the standard model (see figure 3) (see figure 5 in Mann \& Warmuth 2011).

by means of time-of-flight measurements by Burst and Transition Source Experiment (BATSE) onboard the Compton Gamma Ray Observatory (Aschwanden, Schwartz \& Alt 1995b; Aschwanden et al. 1996). Note that the location of the acceleration site is well below the location of the X-point and well above the loop top. Thus, the inflow region of all electrons, which finally travel towards the chromosphere, is typically located in the height range 20-60 Mm above the photosphere. At $40 \mathrm{Mm}$, an electron number density of $2.07 \times 10^{9} \mathrm{~cm}^{-3}$ (see (2.1)) and a magnetic field strength of $46 \mathrm{G}$ (see (2.2)) as well as an Alfvén velocity of $2200 \mathrm{~km} \mathrm{~s}^{-1}$ are obtained as typical plasma parameters in the inflow region (Mann \& Warmuth 2011). Note that these values should be considered as rough ones resulting from a statistical analysis of different flares. From RHESSI measurements, the flux of energetic electrons and the associated energy flux from the reconnection region are known. With this knowledge, Mann \& Warmuth (2011) derived the conditions in the acceleration region by means of the conservation of the total electron number and energy. For large flares, the required huge flux $\left(\approx 10^{36} \mathrm{~s}^{-1}\right)$ of energetic electrons can only be delivered if the inflow speed is nearly equal to the high local Alfvén speed of $\approx 2000-3000 \mathrm{~km} \mathrm{~s}^{-1}$. Recently, Wang, Sui \& Qiu (2007) reported on fast plasma flows with speeds of $900-3500 \mathrm{~km} \mathrm{~s}^{-1}$ as signatures of magnetic reconnection in the corona. As a result, large flares can only occur in region with a high Alfvén speed.

\section{Electron acceleration mechanisms}

NASA's space mission RHESSI (Lin et al. 2002) has the aim to study high-energy processes during solar flares. RHESSI observations require an answer to the question how such a large number of $\approx 10^{36}$ electrons are accelerated up to energies $>30 \mathrm{keV}$ 
within a fraction of a second during large flares. Since these electrons carry a substantial part of the flare released energy (Lin \& Hudson 1971, 1976; Emslie et al. 2004), the answer to this question plays an important role in the understanding of flares.

The reconnection region consists of the inflow, diffusion and outflow regions. The slow-mode shocks separate the inflow region from the outflow one (see figure 3 ). In the framework of the standard model, there are several ideas about where and in which way electrons can be accelerated (for reviews see Vrsnak 2003; Zharkova et al. 2011):

(i) In the diffusion region, magnetic islands can occur in the current sheet. These islands contract and merge with each other. Hence, they can be the source of energetic electrons (Drake et al. 2006; Bárta et al. 2011). Furthermore, a largescale DC electric field is induced within the diffusion region. Electrons can be directly accelerated in this electric field, as studied by Speiser (1984), Speiser \& Lyons (1984), Holman (1985), Benz (1987), Litvinenko \& Somov (1993) and Litvinenko (2000), for instance. This process can even explain the acceleration of an electron up to high energies, but it is not able to provide a high production rate of energetic electrons of typically $10^{36} \mathrm{~s}^{-1}$ as required by the observations because the diffusion region is spatially very small.

(ii) In the outflow region, the newly reconnected magnetic field lines represent a collapsing magnetic trap in the corona. In such collapsing traps, electrons can be accelerated, as studied by Somov \& Kosugi (1997), Karlický \& Kosugi (2004) and Grady \& Neukirch (2009).

(iii) In both the diffusion and outflow regions, the plasma is highly turbulent. Thus, electrons can be accelerated by stochastic acceleration via wave-particle interaction there (Melrose 1994; Miller, Larosa \& Moore 1996; Miteva \& Mann 2007; Petrosian \& Chen 2010; Guo et al. 2012; Chen \& Petrosian 2013; Fleishman \& Toptygin 2013; Bian et al. 2014). The mechanism of stochastic electron acceleration can explain a lot of the observations related to hard X-ray emission during flares.

(iv) If the outflow jet becomes super-Alfvénic, a fast magnetosonic shock, usually called TS, is established due to the interaction of the jet with the surrounding coronal plasma. Signatures of such shocks are readily seen in the solar radio burst radiation (Aurass, Vršnak \& Mann 2002; Aurass \& Mann 2004). The spatial extension of these shocks is assumed to be roughly the width of the loop-top hard X-ray sources first observed by Masuda et al. (1994), i.e. $\approx 7000 \mathrm{~km}$ (Tsuneta et al. 1997). The spatial extent of the TS is much greater than that of the diffusion region, and hence many more electrons can be accelerated at such shocks than in the diffusion region. Therefore, Tsuneta \& Naito (1998) proposed that this shock could be the source of energetic electrons. This mechanism was studied in a quantitative manner for the production of energetic electrons (i.e. $>30 \mathrm{keV}$ ) by Mann, Aurass \& Warmuth (2006) and Mann et al. (2009).

(v) In the standard model, the slow-mode shocks separate the inflow region from the outflow one. At these shocks, magnetic field energy inflowing towards the reconnection region is transformed into heating of the plasma in the downstream region (Tsuneta et al. 1997). That happens efficiently in regions where the Alfvén velocity is extremely large in comparison to the sound speed (Cargill \& Priest 1982). That is even the case in the solar corona. Thus, the heating of the plasma at the slow-mode shocks provides enough electrons with energies beyond $30 \mathrm{keV}$ as required by RHESSI observations (Mann et al. 2014). 
In $\S 3.1$, the acceleration of electrons at the TS is presented in more detail. The role played by the slow-mode shocks for the production of energetic electrons is discussed in $\$ 3.2$.

\subsection{Electron acceleration at the TS}

It is well known that shocks are able to accelerate particles up to high energies in space. Hence, they are considered as the source of cosmic rays (Fermi 1949; Axford, Leer \& Skadron 1978; Schlickeiser 1984; Kirk 1994). Therefore, Tsuneta \& Naito (1998) proposed that the TS in the outflow region is the source of energetic electrons needed for the generation of hard X-ray radiation during flares.

If the speed of the outflow plasma becomes super-Alfvénic, a shock wave is established. An obstacle such as, for example, an underlying loop is not necessary for that. If a super-Alfvénic flow (or jet) is penetrating into the surrounding plasma, it is decelerated owing to the jet-plasma interaction. The transition of a super-Alfvénic flow into a sub-Alfvénic one can basically happen only in terms of a shock wave (see e.g. Priest 1982), which is a dissipative discontinuity. Hence, the TS is a fast-mode shock.

Such shocks are accompanied by a compression of both the density and the magnetic field (see e.g. Priest 1982). Hence, they represent moving magnetic mirrors at which charged particles can be reflected and accelerated. The acceleration happens with the electric field induced in the shock transition region. This mechanism is usually called shock drift acceleration (Holman \& Pesses 1983; Leroy \& Mangeney 1984; Wu 1984). In this section, we consider the TS to be the source of energetic electrons as originally proposed by Tsuneta \& Naito (1998). Since hard $\mathrm{X}$-ray radiation with energies $>100 \mathrm{keV}$ is emitted during flares (Lin \& Hudson 1976), electrons with energies $>100 \mathrm{keV}$ must be produced. Then, the shock drift acceleration of electrons at the TS must be treated in a fully relativistic manner as done by Mann et al. (2006).

Following the approach by Ball \& Melrose (2001) and Mann \& Klassen (2005), the shock drift acceleration is treated in the de Hoffmann-Teller frame, which is defined by removing the motional electric field. Note that the shock is also at rest in this frame. Then, the reflection process can be described by taking into account the conservation of energy and magnetic moment of the particle. Note that all these transformations must necessarily be Lorentzian ones, in contrast to the non-relativistic approach. Doing that, relationships between the velocity components parallel $V_{\|}$and perpendicular $V_{\perp}$ to the upstream magnetic field before $(i)$ and after $(r)$ the reflection are obtained:

$$
\beta_{r, \|}=\frac{2 \beta_{s}-\beta_{i, \|}\left(1+\beta_{s}^{2}\right)}{1-2 \beta_{i, \|} \beta_{s}+\beta_{s}^{2}}
$$

and

$$
\beta_{r, \perp}=\frac{\left(1-\beta_{s}^{2}\right)}{1-2 \beta_{i, \|} \beta_{s}+\beta_{s}^{2}} \beta_{i, \perp},
$$

with $\beta_{\|}=V_{\|} / c$ and $\beta_{\perp}=V_{\perp} / c$ (Mann et al. 2006), respectively. Here, if $v_{s}$ represents the shock speed in the laboratory frame, then $\beta_{s}=v_{s} \sec \theta_{1} / c$ denotes the shock speed in the de Hoffmann-Teller frame, i.e. the shock speed projected onto the magnetic field line, and $\theta_{1}$ is the angle between the shock normal and the upstream magnetic field. Note that here all velocities are normalized to the velocity of light $c$. 


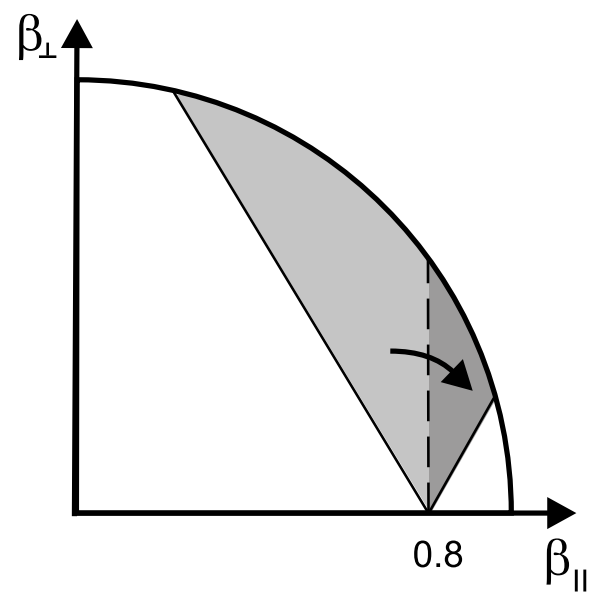

FIGURE 7. Illustration of the shock drift acceleration in the $\beta_{\perp}-\beta_{\|}$plane for a shock wave with $\beta_{s}=0.8, B_{2} / B_{1}=2$ and $\alpha_{l c}=45^{\circ}$ (see figure 2 in Mann et al. 2009).

Additionally, the reflection conditions

$$
\beta_{i, \|} \leqslant \beta_{s}
$$

and

$$
\beta_{i, \perp} \geqslant \frac{\tan \alpha_{l c}}{\sqrt{1-\beta_{s}^{2}}}\left(\beta_{s}-\beta_{i, \|}\right)
$$

must be fulfilled by the initial particles in order to be accelerated. The first one means that the shock must be able to touch a particle in the upstream region. The second one results from the conditions of conservation of the magnetic moment. The loss-cone angle $\alpha_{l c}$ is defined by $\alpha_{l c}=\arcsin \left[\left(B_{1} / B_{2}\right)^{1 / 2}\right]$ with $B_{1}$ and $B_{2}$ as the magnetic field strength in the up- and downstream regions, respectively. Note that this treatment of the shock drift acceleration has been done independently on the charge and mass of the particle. Hence, the results are valid for both electrons and protons.

In the non-relativistic limit, i.e. $\beta_{\|}, \beta_{\perp}, \beta_{s} \ll 1,(3.1)$ and (3.2) reduce to $V_{r, \|}=$ $2 v_{s} \sec \theta_{1}-V_{i, \|}, V_{r, \perp}=V_{i, \perp}$ and $V_{i \perp} \geqslant\left(v_{s} \sec \theta_{1}-V_{i, \|}\right) \tan \alpha_{l c}$ (Holman \& Pesses 1983; Ball \& Melrose 2001; Mann \& Klassen 2005). For example, assuming $10 \times 10^{6} \mathrm{~K}$ as a typical temperature in the flare region (Aschwanden 2005), the electrons have a thermal velocity of $12300 \mathrm{~km} \mathrm{~s}^{-1}$ there. Radio observations reveal $1500 \mathrm{~km} \mathrm{~s}^{-1}$ as a typical speed of the TS (Aurass et al. 2002; Aurass \& Mann 2004). Then, the velocity gain $\Delta V=2 v_{s} \sec \theta_{1}$ due to shock drift acceleration exceeds the one-, four- and 10-fold thermal speed for $\theta_{1}=75.9^{\circ}, 86.5^{\circ}$ and $88.6^{\circ}$, respectively. That demonstrates that shock drift acceleration becomes very efficient if $\theta_{1} \rightarrow 90^{\circ}$, i.e. in the case of nearly perpendicular shocks.

The shock drift acceleration actually represents a transformation in the $\beta_{\perp}-\beta_{\|}$plane as illustrated in figure 7. The initial particles located in the grey area left of the dashed line are transformed into the dark one to the right of it due to shock drift acceleration, as indicated by the arrow in figure 7. This transformation leads to both a gain of energy of each individual particle and an enhancement of the particle number density at high energies in the phase space. That even means particle acceleration. 
As seen in figure 7, the resulting distribution function of the accelerated electrons is a shifted loss-cone distribution as in the non-relativistic case (Leroy \& Mangeney 1984; Wu 1984). In the upstream region a Maxwellian distribution function is assumed for the initial state

$$
f_{M}\left(\beta_{\|}, \beta_{\perp}\right)=C_{M} \mathrm{e}^{-E_{k i n} / k_{B} T}
$$

( $k_{B}$ is Boltzmann's constant and $T$ is temperature), where the temperature $T_{1}$ in the upstream region has to be used in this case. Here, the kinetic energy $E_{k i n}$ is defined by

$$
E_{k i n}=m_{e} c^{2}\left[\frac{1}{\sqrt{1-\beta^{2}}}-1\right]
$$

with the electron mass $m_{e}$ and $\beta^{2}=\beta_{\|}^{2}+\beta_{\perp}^{2}$. Since all particles that fulfill the reflection conditions (see (3.3) and (3.4)) are finally accelerated, the distribution function of the reflected and accelerated particles is derived as

$$
f_{a c c}=C_{M} \Theta\left(\beta_{\|}-\beta_{s}\right) \Theta\left(\beta_{\perp}-D\left[\beta_{s}-\beta_{\|}\right]\right) \exp \left[\frac{(-1)}{\epsilon_{t h}}\left(\frac{1-2 \beta_{s} \beta_{\|}+\beta_{s}^{2}}{\left(1-\beta_{s}^{2}\right)\left(1-\beta^{2}\right)^{1 / 2}}-1\right)\right],
$$

with $D=\tan \alpha_{l c} /\left(1-\beta_{s}^{2}\right)^{1 / 2}, \epsilon_{t h}=k_{B} T_{1} / m_{e} c^{2}$ and $\epsilon=E_{k i n} / m_{e} c^{2}$ (Mann et al. 2009).

As already mentioned in $\S 2.2$ the hard X-ray spectra as measured with RHESSI, for instance, can be converted into a differential flux spectrum of energetic electrons emitting hard X-ray radiation. Hence, the differential flux of energetic electrons is the quantity that can link the theoretical results with the observations.

In a magnetized plasma, as in the corona, the electrons can only propagate along the magnetic field lines over long distances. Hence, they follow the magnetic field lines from the reconnection region in the corona to the dense chromosphere, where they can emit X-ray radiation by bremsstrahlung (Brown 1971). Therefore, the magnetic field-aligned flux of electrons is the quantity of interest. It is generally defined by

$$
\Phi_{\|}=N \int \mathrm{d} \boldsymbol{p} \cdot \boldsymbol{V}_{\|} f
$$

Note that the velocity distribution function is normalized to unity here, and $N$ denotes the particle number density. The particle velocity $\boldsymbol{V}=\boldsymbol{\beta} c$ and its momentum $\boldsymbol{p}$ are related by

$$
\boldsymbol{p}=\frac{m_{e} c \boldsymbol{\beta}}{\sqrt{1-\beta^{2}}} \quad \text { and } \quad \boldsymbol{\beta}=\frac{\boldsymbol{p}^{\prime}}{\sqrt{1+p^{\prime 2}}} .
$$

With $p_{\|}^{\prime}$ and $p_{\perp}^{\prime}$ as the normalized momentum $\left(\boldsymbol{p}^{\prime}=\boldsymbol{p} / m_{e} c\right)$ parallel and perpendicular to the magnetic field, respectively, the magnetic field-aligned flux (see (3.8)) can be written as

$$
\Phi_{\|}=2 \pi N c\left(m_{e} c\right)^{3} \int_{0}^{\infty} \mathrm{d} p_{\|}^{\prime} \mathrm{d} p_{\perp}^{\prime} p_{\perp}^{\prime} \frac{p_{\|}^{\prime}}{\sqrt{1+p^{2}}} f
$$

with $p^{\prime}=\left(p_{\|}^{\prime 2}+p_{\perp}^{\prime 2}\right)^{1 / 2}$ and/or

$$
\Phi_{\|}=2 \pi N c\left(m_{e} c\right)^{3} \int_{0}^{1} \mathrm{~d} \beta_{\|} \mathrm{d} \beta_{\perp} \frac{\beta_{\|} \beta_{\perp}}{\left(1-\beta^{2}\right)^{5 / 2}} f .
$$


In the case of an isotropic distribution function, as for a Maxwellian one, for example, the integral in (3.11) can be treated by means of polar coordinates $\left(p_{\|}^{\prime}=p^{\prime} \cos \alpha\right.$ and $p_{\perp}^{\prime}=p^{\prime} \sin \alpha$ ) with pitch angle $\alpha$. The integration with respect to pitch angle $\alpha$ must be performed from 0 to $\pi / 2$. As a result, one gets an expression for the magnetic field-aligned flux of electrons in terms of an integral with respect to $\epsilon$ :

$$
\Phi_{\|}=\pi N c\left(m_{e} c\right)^{3} \int_{0}^{\infty} \mathrm{d} \epsilon \epsilon(2+\epsilon) f .
$$

The differential flux is generally defined by

$$
j_{\|}\left(E_{k i n}\right)=\frac{\mathrm{d} \Phi_{\|}}{\mathrm{d} E_{k i n}}=\frac{\mathrm{d} \epsilon}{\mathrm{d} E_{k i n}} \frac{\mathrm{d} \Phi_{\|}}{\mathrm{d} \epsilon}=\frac{1}{m_{e} c^{2}} \frac{\mathrm{d} \Phi_{\|}}{\mathrm{d} \epsilon} .
$$

For an example, in the case of a Maxwell distribution (see (3.5)), the normalization constant $C_{M}$ is determined to be

$$
\frac{1}{C_{M}}=4 \pi\left(m_{e} c\right)^{3} \int_{0}^{\infty} \mathrm{d} \epsilon \sqrt{\epsilon(2+\epsilon)}(1+\epsilon) \mathrm{e}^{-\epsilon / \epsilon_{t h}} .
$$

Then, the differential flux of a pure Maxwellian distribution can be derived to be

$$
j_{\|}=\frac{N c}{m_{e} c^{2}} \pi C_{M} \epsilon(2+\epsilon) \mathrm{e}^{-\epsilon / \epsilon_{t h}}
$$

from (3.5), (3.12) and (3.14).

The differential flux of the electrons accelerated by shock drift acceleration can be calculated by inserting the distribution function (3.7) into (3.11) and subsequently performing the integration with respect to $\beta_{\|}$and $\beta_{\perp}$. That was done by Mann et al. (2009). In figure 8 , these fluxes are shown for several angles $\theta$ using typical parameters of the TS, i.e. a shock speed $v_{s}=1500 \mathrm{~km} \mathrm{~s}^{-1}$ (Aurass et al. 2002; Aurass \& Mann 2004) and a temperature $T_{1}=10 \times 10^{6} \mathrm{~K}$ in the flare (Aschwanden 2005). For comparison, the flux of a pure Maxwellian distribution of the same temperature is also presented in figure 8. Figure 8 demonstrates that the fluxes of the accelerated electrons greatly exceed that of a pure Maxwellian population at high energies. The differential fluxes of the accelerated electrons have a local maximum, which shifts towards higher energies with increasing angle $\theta_{1}$. But the flux at the maximum decreases strongly with increasing angle $\theta_{1}$. Those are typical properties of shock drift acceleration.

In figure 8, a Maxwellian distribution has been assumed as the initial one in the upstream region. However, in space plasmas, electron distribution functions are not usually Maxwellian but show an enhanced super-thermal tail (Lin et al. 1996). They are usually described by kappa distributions

$$
f_{\kappa}=C_{\kappa}\left[1+\frac{E_{k i n}}{\kappa E_{\kappa}}\right]^{-(\kappa+1)}
$$

(see Maksimovic, Pierrard \& Riley 1997; Pierrard, Maksimovic \& Lemaire 1999). The case in which a kappa distribution is the initial one in the upstream region is discussed in detail by Mann et al. (2009).

The efficiency of shock drift acceleration is crucially dependent on the angle $\theta$, as figure 8 shows. The magnetic field geometry at the TS is sketched in figure 9. 


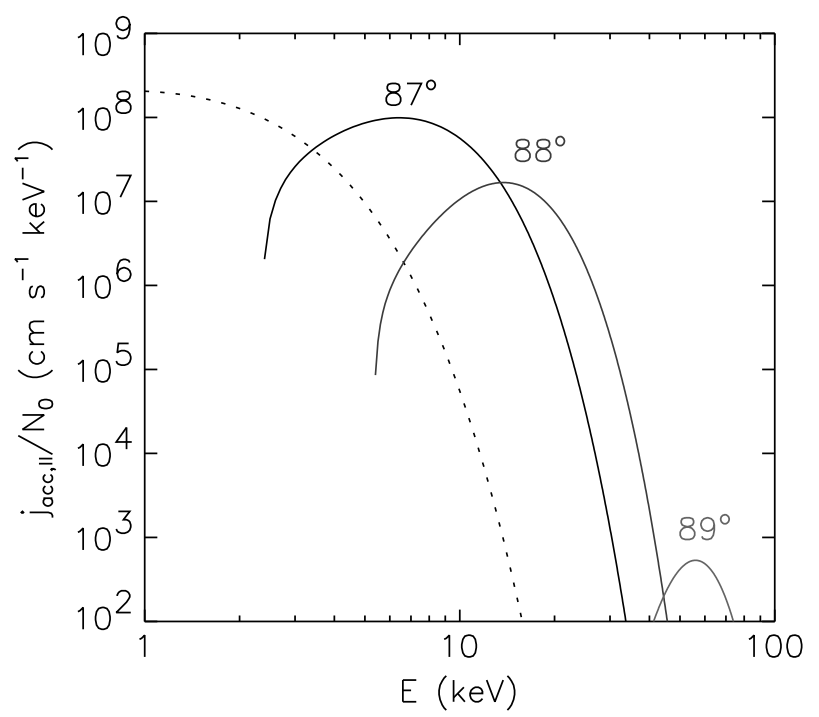

FIGURE 8. The differential magnetic field-aligned flux for electrons accelerated by shock drift acceleration for several angles $\theta_{1}$. Here $N_{0}$ is the electron number density; the shock speed is $1500 \mathrm{~km} \mathrm{~s}^{-1}$; and the loss-cone angle is $45^{\circ}$ according to the jump of the magnetic field $B_{2} / B_{1}=2$ across the shock. For comparison, the corresponding flux of a pure Maxwellian population is presented by the dashed line. A temperature $10 \times 10^{6} \mathrm{~K}$ is adopted in the upstream region (see figure 3 in Mann et al. 2009).

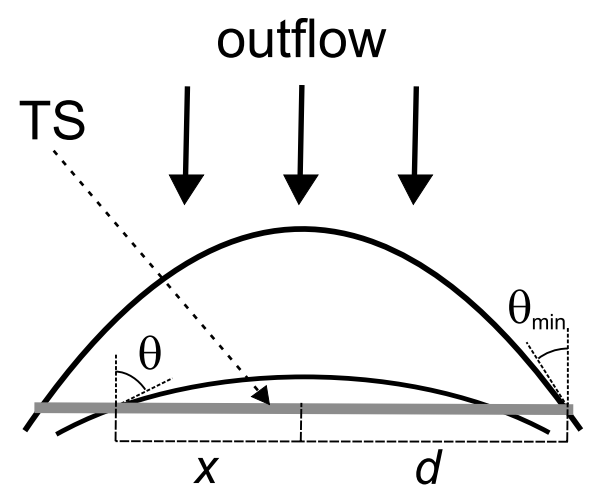

FIGURE 9. Magnetic field geometry at the TS. The reconnected magnetic field lines are pushed across the TS. It takes an angle $\theta$ with the shock normal at the point $x$ (see figure 4 in Mann et al. 2009).

The magnetic field lines are reconnected in the diffusion region (see figure 3) and subsequently convected into the outflow region across the TS. Owing to the curvature of the magnetic field line at the TS, the angle $\theta_{1}$ between the magnetic field and the shock normal varies locally across the shock. Since the shock speed in the de Hoffmann-Teller frame may not exceed the velocity of light, the value of the angle $\theta_{1}$ is limited by $\theta_{1}<\arccos \left(v_{s} / c\right)$, i.e. $\theta_{1}<89.7^{\circ}$ for $v_{s}=1500 \mathrm{~km} \mathrm{~s}^{-1}$. Hence, electrons with different energies are generated at different places at the TS, resulting in a broad spectrum of energetic electrons, as observed. 
At shock drift acceleration, the particles are reflected at the shock by the mirror force and accelerated by the electric field induced in the shock transition region. Basically, the accelerated particles propagate along the magnetic field lines in the upstream region. Owing to the curvature of the magnetic field lines upstream of the TS (see figure 9), they will encounter the shock once again. Since the pitch angle decreases significantly at the first encounter (see (3.2)), the reflection conditions (see (3.4)) are not fulfilled by most of them, so that they can transmit into the downstream region, where they travel along the magnetic field lines towards the chromosphere. There, they emit hard X-rays via bremsstrahlung (Brown 1971).

Warmuth et al. (2009b) discussed whether shock drift acceleration at the TS is able to produce the energetic electrons needed for the hard X-ray emission during flares. For that, the theoretically obtained spectra are compared with the electron spectra derived from hard X-ray spectra measured with RHESSI. Shock drift acceleration can reproduce the total particle and energy fluxes of the electrons with energies beyond the low-energy cut-off, if the plasma in the outflow region is significantly heated and the area of the TS is sufficiently large. The theoretically obtained spectra do not show a true power-law behaviour, but the energy-dependent spectra indices are in the right range (i.e. $3-5)$ up to $\approx 100 \mathrm{keV}$. However, shock drift acceleration at the $\mathrm{TS}$ is able to reproduce the spectral evolution of hard X-ray flare radiation, i.e. the relation between flux and spectral indices: the observed soft-hard-soft and soft-hardharder evolutions of the hard X-ray spectra (Kiplinger 1995; Grigis \& Benz 2004). The observed sub-second hard X-ray bursts can be explained by a variation of the angle $\theta_{1}$ in the framework of this model.

\subsection{Energization of electrons at the slow-mode shocks in the reconnection region}

In contradiction to the fast-mode shocks, at which the kinetic energy of the inflow in the upstream region is transferred to heating of the plasma in the downstream region, at the slow-mode shocks, the inflowing magnetic field energy is transformed to heating of the downstream plasma. Thus, the following question arises. What role does the slow-mode shocks play for the energization of electrons in the reconnection region? That is the subject of this subsection (see Mann et al. (2014)).

The framework of the shock is given in figure 10. The shock normal $\boldsymbol{n}_{s}$ is directed along the $x$ axis, i.e. the shock itself is located in the $y-z$ plane. The magnetic field $\boldsymbol{B}$ is in the $x-z$ plane and takes an angle $\theta_{1}$ to the $x$ axis in the upstream region. As previously, all quantities with subscripts 1 and 2 denote those in the up- and downstream region, respectively.

Since the slow-mode shocks separate the in- and outflow regions, the in- and outflow regions are the up- and downstream ones with respect to the shock transition, respectively. Basically, a shock is a dissipative structure at which quantities such as density, magnetic field and temperature jump, i.e. it is a discontinuity. In the framework of magnetohydrodynamics (MHD), shocks are described by the requirement of the conservation of mass, momentum, energy and magnetic flux across the shock (Priest 1982). These jumps can be expressed in terms of the compression ratio $X=\rho_{2} / \rho_{1}$. They are supplemented by a polynomial of the third order in $X$. Here, the mass density $\rho$ is related to the full particle number density $N$ by $\rho=\tilde{\mu} m_{p} N\left(m_{p}\right.$ is the proton mass and $\tilde{\mu}$ is the mean molecular weight). Additionally, the equation of state $p=N k_{B} T$ ( $p$ is pressure, $T$ is temperature and $k_{B}$ is Boltzmann's constant) has been used. All these equations are called Rankine-Hugoniot relationships. The jump of the magnetic field is found to be

$$
\frac{B_{1}^{2}-B_{2}^{2}}{B_{1}^{2}}=\frac{M a_{A}^{2}}{\left(M a_{A}^{2}-X\right)}(1-X) \sin ^{2} \theta_{1},
$$




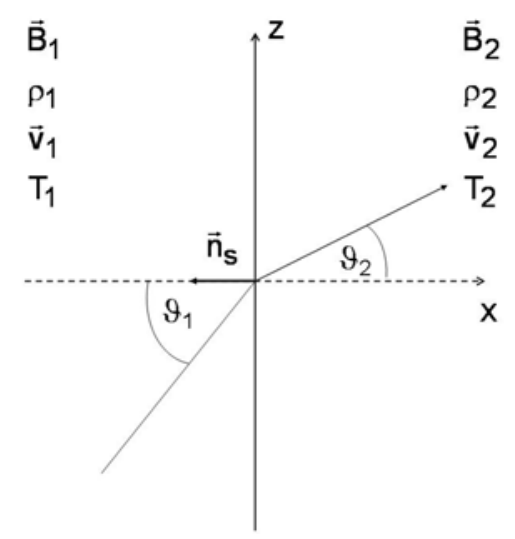

FIgURE 10. The framework of the shock transition. The quantities $\boldsymbol{B}, \rho, \boldsymbol{v}$ and $T$ denote the magnetic field vector, the mass density, the vector of the flow velocity and the temperature in the up- (subscript 1) and downstream (subscript 2) regions, respectively; and $\boldsymbol{n}_{s}$ is the shock normal (see figure 2 in Mann et al. (2014)).

with the Alfvén-Mach number $M a_{A}=\left(v_{s} / v_{A 1}\right) \sec \theta_{1}$. It is actually the Alfvén-Mach number in the de Hoffmann-Teller frame, where the motional electric field is removed. Here, $v_{s}$ denotes the shock speed in the laboratory frame. The Alfvén and sound velocities are defined by $v_{A 1}=B_{1} /\left(4 \pi \rho_{1}\right)^{1 / 2}$ and $c_{s 1}=\left(\gamma p_{1} / \rho_{1}\right)^{1 / 2}$ respectively (where $\gamma$ is the ratio of the specific heats) in the upstream region.

The entropy must increase across the shock, leading to the requirement $X>1$. Then, one gets $M a_{A} \leqslant 1<X$ and $B_{1}>B_{2}$ for the slow-mode shocks. Thus, magnetic field energy is annihilated at the slow-mode shocks and transferred to the heating of the downstream plasma. According to the Rankine-Hugoniot relationships, $T_{2} / T_{1} \propto v_{A 1}^{2} / c_{s 1}^{2}$ is found for the temperature jump across the shock (Priest 1982). Thus, the plasma of the downstream region is strongly heated in regions where the Alfvén speed is extremely high in comparison to the sound speed. That is even the case in flare regions (Mann \& Warmuth 2011) (see §2). That can be explained in the following manner. Because $v_{A 1}^{2} \propto B_{1}^{2} / N_{1}$, there is a large amount of magnetic field energy available per particle in regions with a high Alfvén speed. Since, at the slow-mode shocks, magnetic field energy is transformed into kinetic energy (i.e. heating), that is most efficient in regions where enough magnetic field energy is available per particle, or in other words where the Alfvén velocity is high.

Figure 11 shows the dependence of $T_{2} / T_{1}$ on the Alfvén-Mach number $M a_{A}$ for several angles $\vartheta_{1}$. It can be seen that $T_{2} / T_{1}$ is only weakly dependent on $\theta_{1}$. Inspection of figure 11 reveals that the downstream plasma is strongly heated if $M a_{A}$ approaches 1 and hence $v_{s}=v_{A 1} \cos \theta_{1}, B_{2 z}=0$ and $\theta_{2}=0$. This special case is called a switch-off shock. For this case, the jump in the temperature is given by

$$
\frac{T_{2}}{T_{1}}=1+\frac{(\gamma-1)}{2} \frac{v_{A 1}^{2}}{c_{s 1}^{2}}\left(1-\frac{\cos ^{2} \theta_{1}}{X^{2}}\right) .
$$

Mann et al. (2014) have shown that the transformation of the inflowing magnetic field energy to heating of the downstream plasma is most efficient for $M a_{A}=1$ (i.e. for the switch-off shock) and $\vartheta_{1}=54.7^{\circ}$. Then, the jump of the density is found to 


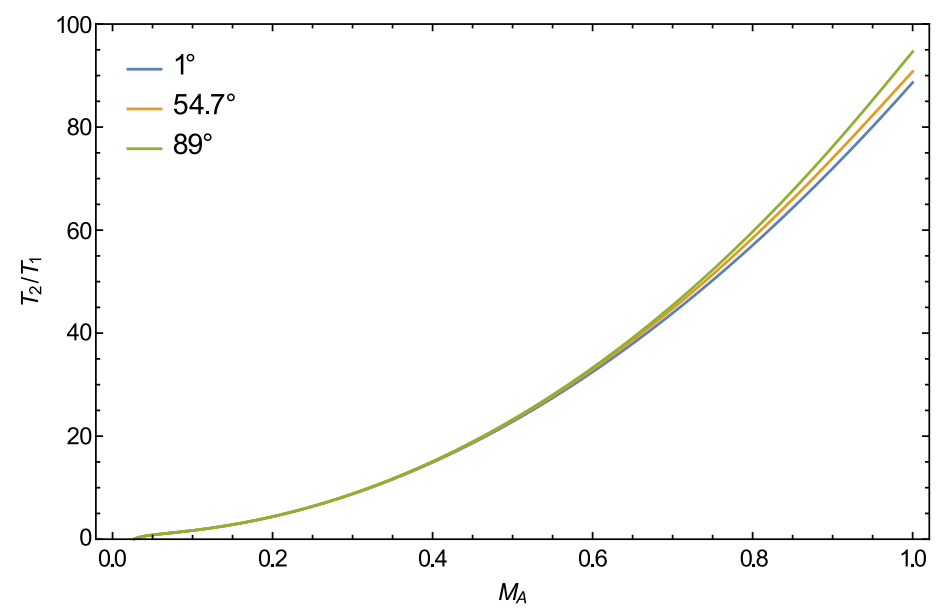

FIGURE 11. Dependence of the temperature jump $T_{2} / T_{1}$ across the slow-mode shock on the Alfvén-Mach number $M a_{A}$ for $\vartheta_{1}=1^{\circ}, 54^{\circ}$ and $89^{\circ}$ with $v_{A 1}=3000 \mathrm{~km} \mathrm{~s}^{-1}$. The sound speed $c_{s 1}=179 \mathrm{~km} \mathrm{~s}^{-1}$ is a typical one in the solar corona for $T_{1}=1.4 \times 10^{6} \mathrm{~K}$ and $\gamma=5 / 3$.

be $\rho_{2} / \rho_{1}=X=2.87$ by means of the numerical evaluation of the Rankine-Hugoniot relationships.

As a result, at slow-mode shocks, magnetic field energy is efficiently annihilated and hence the downstream plasma is strongly energized (Cargill \& Priest 1982; Somov \& Syrovatskii 1982; Forbes \& Malherbe 1991).

Now we will discuss what consequences the slow-mode shocks have for producing energetic electrons during flares (see Mann et al. (2014)). As already mentioned, the electron flux (see (3.8)) parallel to the magnetic field lines is the quantity of interest for comparing the theoretical results with observations. Now, a Maxwellian distribution (see (3.5)) is assumed in the downstream region, where the downstream temperature $T_{2}$ has to be used for the temperature in (3.5)). Then, one gets for the differential flux of electrons along the magnetic field lines (see (3.18))

$$
j_{\|}=\frac{\mathrm{d} \Phi_{\|}}{\mathrm{d} E}=j_{0} \epsilon(2+\epsilon) \mathrm{e}^{-\epsilon / \epsilon_{t h}},
$$

with $j_{0}=\left(N c / m_{e} c^{2}\right) \pi C_{M}\left(m_{e} c\right)^{3}$. It has a local maximum at $\epsilon_{\max }=\epsilon_{t h}-1+\left[1+\epsilon_{t h}^{2}\right]^{1 / 2}$. The maximum shifts towards higher energies for increasing temperatures. For example, it is 0.12 and $3.46 \mathrm{keV}$ for a temperature of $1.4 \times 10^{6}$ and $40 \times 10^{6} \mathrm{~K}$, respectively. By means of (3.19), the flux of electrons with energies $>E_{l c}$ and the related energy flux can be calculated as

$$
\begin{aligned}
F_{e} & =\left(m_{e} c^{2}\right) \int_{\epsilon_{l c}}^{\infty} \mathrm{d} \epsilon j_{\|} \\
& =j_{0} m_{e} c^{2} \mathrm{e}^{-w}\left[2 \epsilon_{t h}^{2}(1+w)+\epsilon_{t h}^{3}\left(2+2 w+w^{2}\right)\right]
\end{aligned}
$$

and

$$
\begin{aligned}
P_{e} & =\left(m_{e} c^{2}\right)^{2} \int_{\epsilon_{l c}}^{\infty} \mathrm{d} \epsilon j_{\|} \epsilon \\
& =j_{0}\left(m_{e} c^{2}\right)^{2} \mathrm{e}^{-w}\left[2 \epsilon_{t h}^{3}\left(2+2 w+w^{2}\right)+\epsilon_{t h}^{4}\left(3+6 w+6 w^{2}+w^{3}\right)\right],
\end{aligned}
$$


with $\epsilon_{l c}=E_{l c} / m_{e} c^{2}$ and $w=\epsilon_{l c} / \epsilon_{t h}$, respectively. In the limit $\epsilon_{t h} \ll 1$, (3.20) and (3.21) provide

$$
\frac{P_{e}}{F_{e}}=\left(m_{e} c^{2}\right) \epsilon_{t h} \frac{\left(2+2 w+w^{2}\right)}{(1+w)} .
$$

In order to compare these theoretical results with observations, GOES X-class flares are considered (see §2.2). This seems to be appropriate since they are the largest ones and the strongest heating of the downstream plasma occurs at the switch-off slow-mode shocks. For these flares, the flux $F_{X C F}$ of electrons with energies $>28 \mathrm{keV}$ and associated energy flux $P_{X C F}$ are $F_{X C F}=3.1 \times 10^{18} \mathrm{~cm}^{-2} \mathrm{~s}^{-1}$ and $P_{e}=P_{X C F}=1.2 \times 10^{20} \mathrm{keV} \mathrm{cm}^{-2} \mathrm{~s}^{-1}$ (see $\S 2.2$ ). If $F_{e}$ and $P_{e}$ are identified with $F_{X C F}$ and $P_{X C F}$, respectively, inspection of (3.20) and (3.21) provides $\epsilon_{t h}=0.0187$ corresponding to a temperature $T_{2}=1.11 \times 10^{8} \mathrm{~K}$ and $N_{2}=8.877 \times 10^{9} \mathrm{~cm}^{-3}$. Note that they are the values of the temperature and the particle number density in the downstream plasma. Here, $\epsilon_{l c}=0.0547$ has been used because $E_{l c}=28 \mathrm{keV}$. In figure 12, the theoretically obtained differential electron flux (see (3.20)) for these values is compared with the measured one (see (2.3)). It shows that the theoretically obtained flux agrees well with the observed ones in the energy range 30-100 keV. Beyond $100 \mathrm{keV}$, the theoretical flux decreases faster than the observed ones, because it does not show a power-law behaviour. The reason for that is that a pure Maxwellian distribution is assumed for the downstream heated electrons. It may be that the electrons show basically a kappa distribution (Lin et al. 1996; Maksimovic et al. 1997; Pierrard et al. 1999) in collisionless plasmas as in the solar corona. Then, a power-law behaviour would result from the heating at the slow-mode shocks. Nevertheless, the energization of electrons at the slow-mode shocks can well reproduce the observed particle fluxes and the associated energy fluxes of flare-generated electrons with energies beyond $30 \mathrm{keV}$. Therefore, the energization at the slow-mode shocks could be a potential mechanism for generating the energetic electrons needed for the hard X-ray radiation during flares.

Now, the plasma parameters in the upstream (or inflow) region can be deduced. Then, $N_{1}=N_{2} / X=8.877 \times 10^{9} \mathrm{~cm}^{-3}$ (because $X=2.87$ ) is the particle number density in the upstream region. For the switch-off shock, (3.18) reduces to $T_{2} / T_{1}=$ $1+0.32\left(v_{A 1}^{2} / c_{s 1}^{2}\right)$. Here, $X=2.87, \theta_{1}=54.7^{\circ}$ and $\gamma=5 / 3$ have been used. Then, one gets $v_{A 1}=2800 \mathrm{~km} \mathrm{~s}^{-1}$ for the Alfvén velocity in the upstream plasma. Here, a typical coronal temperature $T_{1}=1.4 \times 10^{6} \mathrm{~K}$ provides a sound speed $c_{s 1}=179 \mathrm{~km} \mathrm{~s}^{-1}$. This value of the Alfvén speed leads to a magnetic field strength $B_{1}=27 \mathrm{G}$ in the upstream region. This is a typical value for the magnetic field above active regions in the corona (Dulk \& McLean 1978). As a result, $8.9 \times 10^{9} \mathrm{~cm}^{-3}, 1.4 \times 10^{6} \mathrm{~K}$ and $27 \mathrm{G}$ are typical values for the electron number density, temperature and magnetic field strength in regions where large flares can occur. These are coronal regions with large Alfvén velocities of about $2800 \mathrm{~km} \mathrm{~s}^{-1}$.

Adopting these values, $15 \%$ of the electrons inflowing towards the reconnection region are accelerated up to energies beyond $28 \mathrm{keV}$ at the slow-mode shocks. They carry $32 \%$ of the flare-released energy. That agrees well with RHESSI observations (Oka et al. 2013; Krucker \& Battaglia 2014), namely that a substantial part of the flare-released energy is transferred to the energetic electrons. As a result, the energization of electrons at the slow-mode shocks in the magnetic reconnection region provides enough electrons with energies beyond $28 \mathrm{keV}$ as needed for the observed hard X-ray flare emission. 


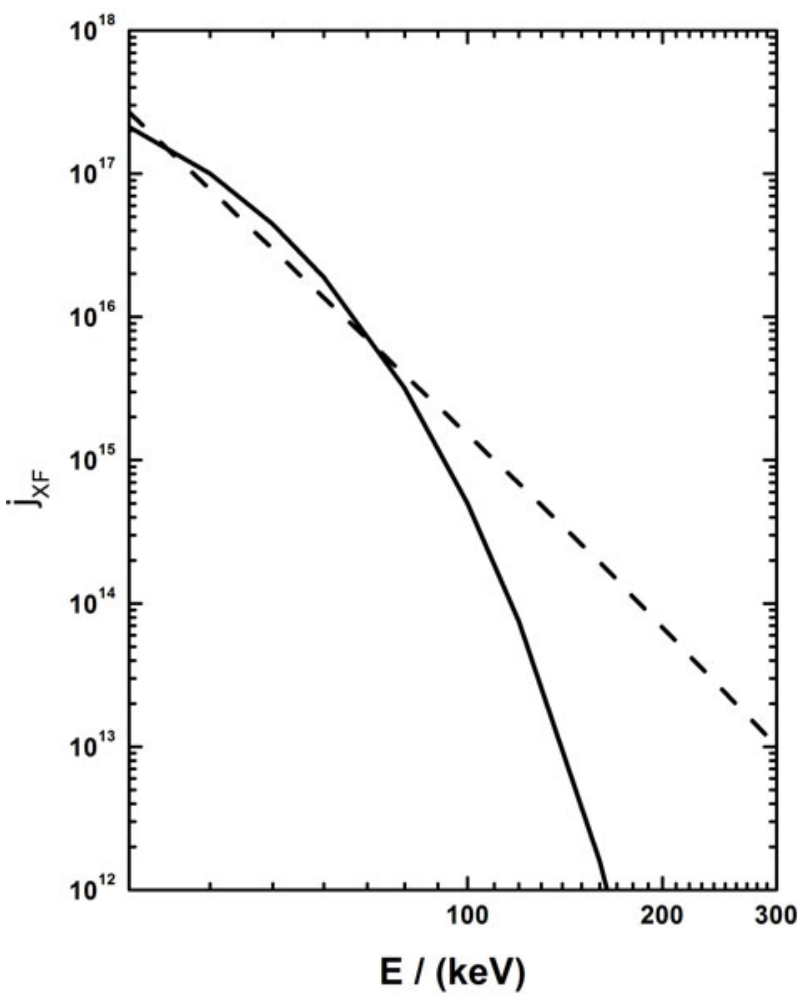

FIGURE 12. Theoretically obtained differential electron fluxes (see (3.19)) (full line) in comparison with such ones measured by RHESSI (see (2.3)) (dashed line).

\section{Summary}

Observations of the hard X-ray emission during flares, as done by NASA's RHESSI spacecraft mission, reveal that a substantial part of the electrons are accelerated up to high energies (i.e. beyond $30 \mathrm{keV}$ ). The spectrum of these electrons can be described in terms of a power law (see $\S 2$ ). The energetic electrons carry a substantial part of the flare-released energy. For this reason, the study of electron acceleration plays an important role in understanding flares. The study of the hard X-ray emission of large (i.e. GOES X-class) flares reveals that large flares occur in regions where the local Alfvén velocity is high, i.e. it must be beyond $\approx 2500 \mathrm{~km} \mathrm{~s}^{-1}$.

Presently, flares are understood in the framework of the standard model (see $\S 1$ ), in which a flare is considered as a manifestation of magnetic reconnection in the solar corona. Several mechanisms of electron acceleration (see $\S 3$ ) have been proposed. They act at different places in the reconnection region, e.g. in the diffusion region, in the turbulent outflow region and at the TS as well as at the slow-mode shocks. The roles that both shocks play for generation of energetic electrons are discussed in detail in $\S \S 3.1$ and 3.2. It was shown that shock drift acceleration at the TS provides enough energetic electrons as needed for the hard X-ray radiation if the outflow plasma in the upstream region of the TS is already sufficiently preheated. In the case of the slow-mode shocks, they generate enough energetic electrons if the Alfvén velocity in the inflow region of the reconnection site is high, i.e. $>2800 \mathrm{~km} \mathrm{~s}^{-1}$. Then, $\approx 15 \%$ of the inflowing electrons are accelerated up to energies $>30 \mathrm{keV}$ and they carry $\approx 35 \%$ 
of the flare-released energy. These results are in good agreement with the observations. But the spectrum of these electrons does not show a power-law behaviour. The reason for that is that a Maxwellian distribution with an exponential decay is assumed for the electrons in the initial state, i.e. before the acceleration process. As already mentioned, so-called kappa distributions with a power-law decay in the supra-thermal tail are usually found for electrons in space plasmas. Adopting such a distribution as the initial one, then a power-law spectrum can result for the energetic electrons from shock drift acceleration at the TS. But, it is also possible that the electrons energized at the slow-mode shocks enter into the outflow region, where they are accelerated subsequently at the TS. Thus, electrons with very high energies can be produced.

Of course, stochastic acceleration of electrons is presently suggested to be the 'best' one to describe the observations of hard X-ray emission during flares (Petrosian \& Chen 2010; Guo et al. 2012; Chen \& Petrosian 2013; Fleishman \& Toptygin 2013). Recently, Bian et al. (2014) reported on a mechanism for the formation of a kappa distribution of flare-accelerated electrons. Owing to diffusive acceleration and Coulomb collisions in the flare region, the resulting electron distribution function approaches asymptotically towards a kappa distribution during its temporal evolution. As already emphasized, RHESSI observations vigorously support the standard (or CSHKP) model. According to this model, there are several sites (e.g. diffusion region, slow- and fast-mode shocks, highly turbulent outflow region) in the reconnection region where electrons can be accelerated by different mechanisms. Since several mechanisms of electron acceleration during flares are possible, there will not be a dominant one. In reality, there will be a mixture and/or combination of all these possible mechanisms, leading to the wealth of phenomena observed in the hard X-ray and $\gamma$-ray emission during solar flares.

The result is that electron acceleration plays an important role for flare physics. In the past, several mechanisms have been proposed. They can explain different facets of the production of energetic electrons during flares. However, there is no complete theoretical model that can explain all the observations. Thus, electron acceleration during solar flares is still an open problem in solar physics.

\section{Acknowledgements}

This work was supported by the German Space Agency 'Deutsches Zentrum für Luft und Raumfahrt' under grant no. 50 QL 0901. The author is grateful to the RHESSI team (PI R. P. Lin and, presently, S. Krucker) for free access to the RHESSI data. In particular, the author expresses his thanks to his colleagues, H. Aurass, S. Braune, F. Breitling, D. Lehmann, R. Miteva, H. Önel, C. Vocks and A. Warmuth for the fruitful collaboration with them in the past decades.

\section{REFERENCES}

Aschwanden, M. J. 2002 Particle acceleration and kinematics in solar flares - a synthesis of recent observations and theoretical concepts. Space Sci. Rev. 101, 1-227.

Aschwanden, M. J. 2005 Physics of the Solar Corona: An Introduction with Problems and Solutions, 2nd edn. Praxis.

Aschwanden, M. J., Benz, A. O., Dennis, B. R. \& Schwartz, R. A. 1995a Solar electron beams detected in hard x-rays and radio waves. Astrophys. J. 455, 347-365.

Aschwanden, M. J., Kosugi, T., Hudson, H. S., Wills, M. J. \& Schwartz, R. A. 1996 The scaling law between electron time-of-flight distances and loop lengths in solar flares. Astrophys. J. 470, 1198. 
Aschwanden, M. J., Schwartz, R. A. \& Alt, D. M. $1995 b$ Electron time-of-flight differences in solar flares. Astrophys. J. 447, 923.

Aulanier, G., Janvier, M. \& Schmieder, B. 2012 The standard flare model in three dimensions. I. Strong-to-weak shear transition in post-flare loops. Astron. Astrophys. 543, A110.

Aurass, H. \& MANN, G. 2004 Radio observation of electron acceleration at solar flare reconnection outflow termination shocks. Astrophys. J. 615, 526-530.

AURASS, H., VRŠNAK, B. \& MANN, G. 2002 Shock-excited radio burst from reconnection outflow jet? Astron. Astrophys. 384, 273-281.

AXFord, W. I., LEer, E. \& SKADRON, G. 1978 Acceleration of cosmic rays by shock waves. In Cosmophysics (ed. V. A. Dergachev \& G. E. Kocharov), pp. 125-134.

Ball, L. \& Melrose, D. B. 2001 Shock drift acceleration of electrons. Publ. Astron. Soc. Australia 18, 361-373.

BÁRTA, M., BÜChner, J., KARlickÝ, M. \& SkÁla, J. 2011 Spontaneous current-layer fragmentation and cascading reconnection in solar flares. I. Model and analysis. Astrophys. J. 737, 24.

Battaglia, M. \& BenZ, A. O. 2006 Relations between concurrent hard X-ray sources in solar flares. Astron. Astrophys. 456, 751-760.

Benz, A. O. 1987 Acceleration and energization by currents and electric fields. Solar Phys. 111, $1-18$.

Bian, N. H., Emslie, A. G., Stackhouse, D. J. \& Kontar, E. P. 2014 The formation of kappa-distribution accelerated electron populations in solar flares. Astrophys. J. 796, 142.

BRown, J. C. 1971 The deduction of energy spectra of non-thermal electrons in flares from the observed dynamic spectra of hard x-ray bursts. Solar Phys. 18, 489-502.

Cargill, P. J. \& Priest, E. R. 1982 Slow-shock heating and the Kopp-Pneuman model for 'post'-flare loops. Solar Phys. 76, 357-375.

CARmichael, H. 1964 A process for flares. NASA Spec. Pub. 50, p. 451.

Chen, Q. \& Petrosian, V. 2013 Determination of stochastic acceleration model characteristics in solar flares. Astrophys. J. 777, 33.

Cliver, E. W. \& Dietrich, W. F. 2013 The 1859 space weather event revisited: limits of extreme activity. J. Space Weather Space Clim. 3 (27), A31.

Cliver, E. W. \& SvalgaARD, L. 2004 The 1859 solar-terrestrial disturbance and the current limits of extreme space weather activity. Solar Phys. 224, 407-422.

Drake, J. F., Swisdak, M., Che, H. \& Shay, M. A. 2006 Electron acceleration from contracting magnetic islands during reconnection. Nature 443, 553-556.

Dulk, G. A. \& Mclean, D. J. 1978 Coronal magnetic fields. Solar Phys. 57, 279-295.

Emslie, A. G., Dennis, B. R., Shih, A. Y., Chamberlin, P. C., Mewaldt, R. A., Moore, C. S., Share, G. H., Vourlidas, A. \& Welsch, B. T. 2012 Global energetics of thirty-eight large solar eruptive events. Astrophys. J. 759, 71.

Emslie, A. G., Kontar, E. P., Krucker, S. \& Lin, R. P. 2003 RHESSI hard x-ray imaging spectroscopy of the large gamma-ray flare of 2002 July 23. Astrophys. J. Lett. 595, L107-L110.

Emslie, A. G., Kucharek, H., Dennis, B. R., Gopalswamy, N., Holman, G. D., Share, G. H., Vourlidas, A., Forbes, T. G., Gallagher, P. T., Mason, G. M. et al. 2004 Energy partition in two solar flare/CME events. J. Geophys. Res. 109 (A18), 10104.

Fermi, E. 1949 On the origin of the cosmic radiation. Phys. Rev. 75, 1169-1174.

Fleishman, G. D. \& Toptygin, I. N. 2013 Stochastic particle acceleration by helical turbulence in solar flares. Mon. Not. R. Astron. Soc. 429, 2515-2526.

Fletcher, L., Dennis, B. R., Hudson, H. S., Krucker, S., Phillips, K., Veronig, A., Battaglia, M., Bone, L., CAspi, A., Chen, Q. et al. 2011 An observational overview of solar flares. Space Sci. Rev. 159, 19-106.

Forbes, T. G. 1986 Fast-shock formation in line-tied magnetic reconnection models of solar flares. Astrophys. J. 305, 553-563.

Forbes, T. G. \& MAlmerbe, J. M. 1991 A numerical simulation of magnetic reconnection and radiative cooling in line-tied current sheets. Solar Phys. 135, 361-391.

Grady, K. J. \& NeUKIRCH, T. 2009 An extension of the theory of kinematic MHD models of collapsing magnetic traps to $2.5 \mathrm{D}$ with shear flow and to 3D. Astron. Astrophys. 508, $1461-1468$. 
Grigis, P. C. \& Benz, A. O. 2004 The spectral evolution of impulsive solar X-ray flares. Astron. Astrophys. 426, 1093-1101.

Guo, J., Emslie, A. G., Kontar, E. P., Benvenuto, F., Massone, A. M. \& Piana, M. 2012 Determination of the acceleration region size in a loop-structured solar flare. Astron. Astrophys. 543, A53.

Heyvaerts, J. 1981 Solar Flare Magnetohydrodynamics. Gordon and Breach.

HiRAYAMA, T. 1974 Theoretical model of flares and prominences. I: Evaporating flare model. Solar Phys. 34, 323-338.

Holman, G. D. 1985 Acceleration of runaway electrons and Joule heating in solar flares. Astrophys. J. 293, 584-594.

Holman, G. D., Aschwanden, M. J., Aurass, H., Battaglia, M., Grigis, P. C., Kontar, E. P., Liu, W., Saint-Hilaire, P. \& Zharkova, V. V. 2011 Implications of x-ray observations for electron acceleration and propagation in solar flares. Space Sci. Rev. 159, $107-166$.

Holman, G. D. \& Pesses, M. E. 1983 Solar type II radio emission and the shock drift acceleration of electrons. Astrophys. J. 267, 837-843.

Holman, G. D., Sui, L., Schwartz, R. A. \& Emslie, A. G. 2003 Electron bremsstrahlung hard $\mathrm{x}$-ray spectra, electron distributions, and energetics in the 2002 July 23 solar flare. Astrophys. J. Lett. 595, L97-L101.

KARLICKÝ, M. \& KosUgi, T. 2004 Acceleration and heating processes in a collapsing magnetic trap. Astron. Astrophys. 419, 1159-1168.

KiplingeR, A. L. 1995 Comparative studies of hard x-ray spectral evolution in solar flares with high-energy proton events observed at Earth. Astrophys. J. 453, 973.

KIRK, J. G. 1994 Particle acceleration. In Saas-Fee Advanced Course 24: Plasma Astrophysics (ed. J. G. Kirk, D. B. Melrose, E. R. Priest, A. O. Benz \& T. J.-L. Courvoisier), p. 225. Springer.

Klein, K.-L. \& Trottet, G. 2001 The origin of solar energetic particle events: coronal acceleration versus shock wave acceleration. Space Sci. Rev. 95, 215-225.

Kontar, E. P., Brown, J. C., Emslie, A. G., Hajdas, W., Holman, G. D., Hurford, G. J., KašParová, J., Mallik, P. C. V., Massone, A. M., MCConnell, M. L. et al. 2011 Deducing electron properties from hard x-ray observations. Space Sci. Rev. 159, 301-355.

Kontar, E. P., MacKinnon, A. L., Schwartz, R. A. \& Brown, J. C. 2006 Compton backscattered and primary X-rays from solar flares: angle dependent Green's function correction for photospheric albedo. Astron. Astrophys. 446, 1157-1163.

Kopp, R. A. \& Pneuman, G. W. 1976 Magnetic reconnection in the corona and the loop prominence phenomenon. Solar Phys. 50, 85-98.

Koutchmy, S. 1994 Coronal physics from eclipse observations. Adv. Space Res. 14, 29.

Krucker, S. \& Battaglia, M. 2014 Particle densities within the acceleration region of a solar flare. Astrophys. J. 780, 107.

Krucker, S., Battaglia, M., Cargill, P. J., Fletcher, L., Hudson, H. S., MacKinnon, A. L., Masuda, S., Sui, L., Tomczak, M., Veronig, A. L. et al. $2008 a$ Hard X-ray emission from the solar corona. Astron. Astrophys. Rev. 16, 155-208.

Krucker, S., HANnAH, I. G. \& Lin, R. P. 2007 RHESSI and HINODE x-ray observations of a partially occulted solar flare. Astrophys. J. Lett. 671, L193-L196.

Krucker, S., Hudson, H. S., Glesener, L., White, S. M., Masuda, S., Wuelser, J.-P. \& Lin, R. P. 2010 Measurements of the coronal acceleration region of a solar flare. Astrophys. J. 714, 1108-1119.

Krucker, S., Hurford, G. J. \& Lin, R. P. 2003 Hard x-ray source motions in the 2002 July 23 gamma-ray flare. Astrophys. J. Lett. 595, L103-L106.

Krucker, S., Hurford, G. J., MacKinnon, A. L., Shih, A. Y. \& Lin, R. P. $2008 b$ Coronal $\gamma$-ray bremsstrahlung from solar flare-accelerated electrons. Astrophys. J. Lett. 678, L63-L66.

KRUCKER, S. \& LIN, R. P. 2008 Hard x-ray emissions from partially occulted solar flares. Astrophys. J. 673, 1181-1187. 
Leblanc, Y., Dulk, G. A. \& Bougeret, J.-L. 1998 Tracing the electron density from the corona to 1 AU. Solar Phys. 183, 165-180.

Leroy, M. M. \& MAngeney, A. 1984 A theory of energization of solar wind electrons by the Earth's bow shock. Ann. Geophys. 2, 449-456.

LIN, R. P. 1974 Non-relativistic solar electrons. Space Sci. Rev. 16, 189-256.

Lin, R. P., Dennis, B. R., Hurford, G. J., Smith, D. M., Zehnder, A., Harvey, P. R., Curtis, D. W., Pankow, D., Turin, P., Bester, M. et al. 2002 The Reuven Ramaty High-Energy Solar Spectroscopic Imager (RHESSI). Solar Phys. 210, 3-32.

LIN, R. P. \& HUdSON, H. S. 1971 10-100 keV electron acceleration and emission from solar flares. Solar Phys. 17, 412-435.

Lin, R. P. \& Hudson, H. S. 1976 Non-thermal processes in large solar flares. Solar Phys. 50, $153-178$.

Lin, R. P., Larson, D., McFadden, J., Carlson, C. W., Ergun, R. E., Anderson, K. A., Ashford, S., MCCARThy, M., PARKs, G. K., RÈme, H. et al. 1996 Observation of an impulsive solar electron event extending down to $\approx 0.5 \mathrm{keV}$ energy. Geophys. Res. Lett. 23, $1211-1214$.

Litvinenko, Y. E. 2000 Electron acceleration by strong DC electric fields in impulsive solar flares. In High Energy Solar Physics Workshop - Anticipating Hess! (ed. R. Ramaty \& N. Mandzhavidze), Astronomical Society of the Pacific Conference Series, vol. 206, p. 167. Astronomical Society of the Pacific.

Litvinenko, Y. E. \& Somov, B. V. 1993 Particle acceleration in reconnecting current sheets. Solar Phys. 146, 127-133.

Maksimovic, M., Pierrard, V. \& Riley, P. 1997 Ulysses electron distributions fitted with kappa functions. Geophys. Res. Lett. 24, 1151-1154.

Mann, G., Aurass, H., Önel, H. \& Warmuth, A. 2014 On the role of slow-mode shocks in the reconnection region for generating energetic electros during solar flares. Astron. Astrophys. (submitted).

ManN, G., Aurass, H. \& WARmuth, A. 2006 Electron acceleration by the reconnection outflow shock during solar flares. Astron. Astrophys. 454, 969-974.

Mann, G., Jansen, F., MacDowall, R. J., Kaiser, M. L. \& Stone, R. G. 1999 A heliospheric density model and type III radio bursts. Astron. Astrophys. 348, 614-620.

MANn, G. \& KlASSEN, A. 2005 Electron beams generated by shock waves in the solar corona. Astron. Astrophys. 441, 319-326.

MANn, G. \& WARMUth, A. 2011 Budget of energetic electrons during solar flares in the framework of magnetic reconnection. Astron. Astrophys. 528, A104.

Mann, G., Warmuth, A. \& Aurass, H. 2009 Generation of highly energetic electrons at reconnection outflow shocks during solar flares. Astron. Astrophys. 494, 669-675.

Massone, A. M., Piana, M., Conway, A.\& Eves, B. 2003 A regularization approach for the analysis of RHESSI X-ray spectra. Astron. Astrophys. 405, 325-330.

Masuda, S., Kosugi, T., Hara, H., Tsuneta, S. \& Ogawara, Y. 1994 A loop-top hard X-ray source in a compact solar flare as evidence for magnetic reconnection. Nature 371, 495-497.

MCLeAn, D. J. 1985 Metre-wave solar radio bursts. In Solar Radiophysics: Studies of Emission from the Sun at Metre Wavelengths (ed. D. J. McLean \& N. R. Labrum), pp. 37-52. Cambridge University Press.

Melrose, D. B. 1985 Plasma emission mechanisms. In Solar Radiophysics: Studies of Emission from the Sun at Metre Wavelengths (ed. D. J. McLean \& N. R. Labrum), pp. 177-210. Cambridge University Press.

Melrose, D. B. 1994 Turbulent acceleration in solar flares. Astrophys. J. Suppl. Ser. 90, 623-630.

Miller, J. A., LarosA, T. N. \& Moore, R. L. 1996 Stochastic electron acceleration by cascading fast mode waves in impulsive solar flares. Astrophys. J. 461, 445.

Miteva, R. \& MAnN, G. 2007 The electron acceleration at shock waves in the solar corona. Astron. Astrophys. 474, 617-625.

NEWKIRK, G. JR. 1961 The solar corona in active regions and the thermal origin of the slowly varying component of solar radio radiation. Astrophys. J. 133, 983. 
Oka, M., Ishikawa, S., Saint-Hilaire, P., Krucker, S. \& Lin, R. P. 2013 Kappa distribution model for hard X-ray coronal sources of solar flares. Astrophys. J. 764, 6.

Petrosian, V. \& Chen, Q. 2010 Derivation of stochastic acceleration model characteristics for solar flares from RHESSI hard x-ray observations. Astrophys. J. Lett. 712, L131-L134.

Piana, M., Massone, A. M., Kontar, E. P., Emslie, A. G., Brown, J. C. \& Schwartz, R. A. 2003 Regularized electron flux spectra in the 2002 July 23 solar flare. Astrophys. J. Lett. 595, L127-L130.

Pierrard, V., MaKsimovic, M. \& Lemaire, J. 1999 Electron velocity distribution functions from the solar wind to the corona. J. Geophys. Res. 104, 17021-17032.

PRIEST, E. R. 1981 Solar flare magnetohydrodynamics. In Solar Flare Magnetohydrodynamics. Gordon and Breach.

PRIEST, E. R. 1982 Solar magneto-hydrodynamics. Geophys. Astrophys. Monographs 21.

Priest, E. R. \& Forbes, T. G. 2002 The magnetic nature of solar flares. Astron. Astrophys. Rev. 10, 313-377.

Reames, D. V., BARbier, L. M. \& NG, C. K. 1996 The spatial distribution of particles accelerated by coronal mass ejection-driven shocks. Astrophys. J. 466, 473.

Reid, H. A. S., Vilmer, N. \& KontaR, E. P. 2011 Characteristics of the flare acceleration region derived from simultaneous hard X-ray and radio observations. Astron. Astrophys. 529, A66.

Reid, H. A. S., Vilmer, N. \& Kontar, E. P. 2014 The low-high-low trend of type III radio burst starting frequencies and solar flare hard X-rays. Astron. Astrophys. 567, A85.

Saito, K., Makita, M., Nishi, K. \& HatA, S. 1970 A non-spherical axisymmetric model of the solar K corona of the minimum type. Ann. Tokyo Astron. Obs. 12, 53-120.

Saito, K., Poland, A. I. \& Munro, R. H. 1977 A study of the background corona near solar minimum. Solar Phys. 55, 121-134.

SCHLICKEISER, R. 1984 An explanation of abrupt cutoffs in the optical-infrared spectra of nonthermal sources - a new pile-up mechanism for relativistic electron spectra. Astron. Astrophys. 136, 227-236.

Shibata, K., Ishido, Y., Acton, L. W., Strong, K. T., Hirayama, T., Uchida, Y., McAllister, A. H., Matsumoto, R., Tsuneta, S., Shimizu, T. et al. 1992 Observations of X-ray jets with the YOHKOH soft X-ray telescope. Publ. Astron. Soc. Japan 44, L173-L179.

Shibata, K., Masuda, S., Shimojo, M., Hara, H., Yokoyama, T., Tsuneta, S., Kosugi, T. \& Ogawara, Y. 1995 Hot-plasma ejections associated with compact-loop solar flares. Astrophys. J. Lett. 451, L83.

Simões, P. J. A. \& KontaR, E. P. 2013 Implications for electron acceleration and transport from non-thermal electron rates at looptop and footpoint sources in solar flares. Astron. Astrophys. 551, A135.

Smith, D. M., Lin, R. P., Turin, P., Curtis, D. W., Primbsch, J. H., Campbell, R. D., Abiad, R., Schroeder, P., Cork, C. P., Hull, E. L. et al. 2002 The RHESSI spectrometer. Solar Phys. 210, 33-60.

Somov, B. V. \& Kosugi, T. 1997 Collisionless reconnection and high-energy particle acceleration in solar flares. Astrophys. J. 485, 859-868.

Somov, B. V. \& SYRovATSKII, S. I. 1982 Thermal trigger for solar flares and coronal loops formation. Solar Phys. 75, 237-244.

SPEISER, T. W. 1984 Current sheet particle acceleration - theory and observations for the geomagnetic tail. Adv. Space Res. 4, 439-448.

SPEISER, T. W. \& LyONS, L. R. 1984 Comparison of an analytical approximation for particle motion in a current sheet with precise numerical calculations. J. Geophys. Res. 89, 147-158.

StURrock, P. A. 1966 Model of the high-energy phase of solar flares. Nature 211, 695-697.

Suzuki, S. \& DulK, G. A. 1985 Bursts of type III and type V. In Solar Radiophysics: Studies of Emission from the Sun at Metre Wavelengths (ed. D. J. McLean \& N. R. Labrum), pp. 289-332. Cambridge University Press.

Treumann, R. A. \& Baumjohann, W. 1997 Advanced Space Plasma Physics. Imperial College Press. 
Tsuneta, S. 1996 Structure and dynamics of magnetic reconnection in a solar flare. Astrophys. J. 456, 840.

Tsuneta, S., Masuda, S., Kosugi, T. \& Sato, J. 1997 Hot and superhot plasmas above an impulsive flare loop. Astrophys. J. 478, 787-798.

TsunetA, S. \& NAito, T. 1998 Fermi acceleration at the fast shock in a solar flare and the impulsive loop-top hard x-ray source. Astrophys. J. Lett. 495, L67-L70.

Vilmer, N., KRUCKer, S., Lin, R. P. \& RHESSI TeAm 2002 Hard x-ray and metric/decimetric radio observations of the 20 February 2002 solar flare. Solar Phys. 210, 261-272.

VRSNAK, B. 2003 Magnetic 3D configurations of energy release in solar flares. In Energy Conversion and Particle Acceleration in the Solar Corona (ed. L. Klein), Lecture Notes in Physics, vol. 612, pp. 28-47. Springer.

WANG, T., SUI, L. \& QIU, J. 2007 Direct observation of high-speed plasma outflows produced by magnetic reconnection in solar impulsive events. Astrophys. J. Lett. 661, L207-L210.

Warmuth, A., Holman, G. D., Dennis, B. R., Mann, G., Aurass, H. \& Milligan, R. O. $2009 a$ Rapid changes of electron acceleration characteristics at the end of the impulsive phase of an X-class solar flare. Astrophys. J. 699, 917-922.

Warmuth, A.\& Mann, G. 2005 A model of the Alfvén speed in the solar corona. Astron. Astrophys. 435, 1123-1135.

Warmuth, A., Mann, G. \& Aurass, H. 2007 Constraining electron acceleration at a standing shock with HXR and radio observations. Cent. Eur. Astrophys. Bull. 31, 135.

Warmuth, A., Mann, G. \& Aurass, H. 2009b Modelling shock drift acceleration of electrons at the reconnection outflow termination shock in solar flares. Observational constraints and parametric study. Astron. Astrophys. 494, 677-691.

White, S. M., Benz, A. O., Christe, S., FÁrník, F., Kundu, M. R., Mann, G., Ning, Z., Raulin, J.-P., Silva-VÁlio, A. V. R., Saint-Hilaire, P. et al. 2011 The relationship between solar radio and hard X-ray emission. Space Sci. Rev. 159, 225-261.

WU, C. S. 1984 A fast Fermi process - energetic electrons accelerated by a nearly perpendicular bow shock. J. Geophys. Res. 89, 8857-8862.

Yokoyama, T. \& ShibatA, K. 1994 Numerical simulation of reconnection between emerging flux and coronal field. In Proceedings of Kofu Symposium, Tokyo 181, Japan, pp. 367-370. National Astronomical Observatory.

Zharkova, V. V., Arzner, K., Benz, A. O., Browning, P., Dauphin, C., Emslie, A. G., Fletcher, L., Kontar, E. P., Mann, G., OnOfRI, M. et al. 2011 Recent advances in understanding particle acceleration processes in solar flares. Space Sci. Rev. 159, 357-420. 